ESAIM: M2AN 46 (2012) 1421-1445

DOI: $10.1051 / \mathrm{m} 2 \mathrm{an} / 2012011$
ESAIM: Mathematical Modelling and Numerical Analysis

www.esaim-m2an.org

\title{
ELECTROMAGNETIC SCATTERING AT COMPOSITE OBJECTS: A NOVEL MULTI-TRACE BOUNDARY INTEGRAL FORMULATION
}

\author{
Xavier Claeys ${ }^{1}$ And Ralf Hiptmair ${ }^{2}$
}

\begin{abstract}
Since matrix compression has paved the way for discretizing the boundary integral equation formulations of electromagnetics scattering on very fine meshes, preconditioners for the resulting linear systems have become key to efficient simulations. Operator preconditioning based on Calderón identities has proved to be a powerful device for devising preconditioners. However, this is not possible for the usual first-kind boundary formulations for electromagnetic scattering at general penetrable composite obstacles. We propose a new first-kind boundary integral equation formulation following the reasoning employed in [X. Clayes and R. Hiptmair, Report 2011-45, SAM, ETH Zürich (2011)] for acoustic scattering. We call it multi-trace formulation, because its unknowns are two pairs of traces on interfaces in the interior of the scatterer. We give a comprehensive analysis culminating in a proof of coercivity, and uniqueness and existence of solution. We establish a Calderón identity for the multi-trace formulation, which forms the foundation for operator preconditioning in the case of conforming Galerkin boundary element discretization.
\end{abstract}

Mathematics Subject Classification. 78A45, 35A35, 35Q60, 78M15.

Received March 13, 2012. Revised March 13, 2012.

Published online May 31, 2012.

\section{INTRODUCTION}

The scattering of electromagnetic waves by penetrable obstacles is of practical interest in many applications. If the obstacle is composed of a few linear homogeneous dielectric media, boundary integral equation methods are an attractive option for solving the scattering problem numerically. Thus, in this article we consider the transmission problems for the linear Maxwell equations in frequency domain of the form

$$
\begin{aligned}
& \operatorname{curl} \operatorname{curl}(\mathbf{u})-\kappa_{j}^{2} \mathbf{u}=\mathbf{0} \text { in } \Omega_{j}, \quad j=0 \ldots n, \\
& + \text { radiation condition at infinity, } \\
& + \text { transmission conditions at interfaces. }
\end{aligned}
$$

Here, $\kappa_{j}$ refers to the wave number in subdomain $\Omega_{j}$, and no particular assumption is imposed on the geometry (except for some minimal regularity of $\partial \Omega_{j}, j=0 \ldots n$ to allow the definition of traces). In such a problem, that

\footnotetext{
Keywords and phrases. Integral equations, boundary element method, domain decomposition, Maxwell's equations.

1 Université de Toulouse, ISAE, 10 Avenue Edouard-Belin, 31055 Toulouse, France. xavier.claeys@isae.fr

2 Seminar of Applied Mathematics, ETHZ, 8092 Zürich, Switzerland. hiptmair@sam.math.ethz.ch
} 


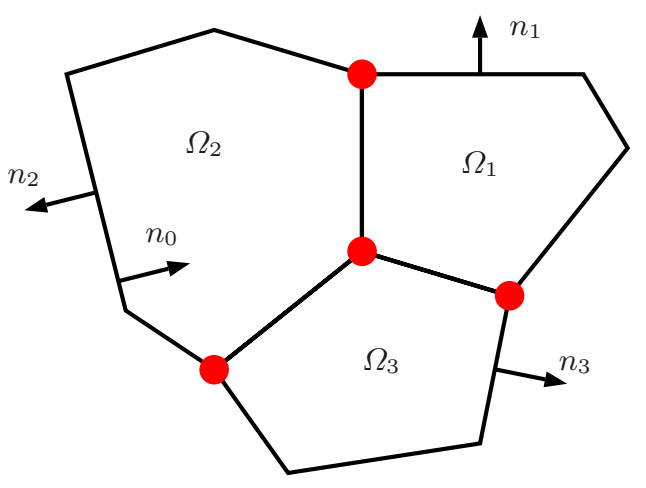

$\Omega_{0}$
$:=$
exterior domain

FigURE 1. Cross-section of an admissible arrangement of three subdomains.

we call multi-subdomain scattering problem, there may be edges where three or more subdomains $\Omega_{j}$ abut, see Figure 1 below.

This very general setting goes beyond what is usually discussed in the literature on boundary integral equation methods for electromagnetics with many works only dealing with homogeneous or impenetrable scatterers. A few approaches address the general situation (1.1), most notably the Poggio-Miller-Chew-Harrington-Wu-Tsai formulation (PMCHWT, $[14,32,40]$ ), whose rigorous mathematical analysis was first accomplished in [6]. The unknowns in this formulation are only one pair of traces at each point of each interface; we can classify it as single-trace formulation. Further, it is not affected by spurious resonances.

However, as boundary integral equations of the first kind after Galerkin boundary element discretization based on the usual surface edge elements (RWG basis functions, [3,33]) the PMCHWT equations spawn illconditioned matrices on fine meshes. This cripples the convergence of iterative solution methods like GMRES, which is serious challenge, because there is no alternative to iterative solvers when matrix compression techniques like multipole are applied to the discrete boundary integral operators.

Thus, preconditioning of the discrete boundary integral equations becomes indispensable and has attracted considerable attention. In particular, a variant of operator preconditioning [25], the so-called Calderón preconditioners introduced in $[16,17,35]$, have briskly been adopted in computational electromagnetism $[2,21,37,41]$.

Unfortunately, no satisfactory Calderón preconditioner for the PMCHWT has been found. This missing preconditioner motivated the present article. The main ideas have first been elaborated for acoustic scattering in [19] and now we adapt them to Maxwell's equations. Again, we start with the observation that the single-trace formulation admits straightforward Calderón preconditioning in the case of scattering at a single homogeneous object $(n=1$ in (1.1)). The general situation can be converted to this special setting by introducing a narrow "virtual air gap" separating the subdomains $\Omega_{j}$. We find that all integral operators remain well defined when we let the width of the air gap tend to zero. This formal procedure yields the new formulation and its associated Calderón preconditioner. We retain the traces on both sides of the air gap as unknowns. Therefore we end up with two pairs of unknown traces on each interior subdomain interface, and we dub the new set of equations "multi-trace".

Ours is not the only multi-trace formulation designed with preconditioning in mind. A similar approach has been proposed in [26], but only for the acoustic case. A related technique, the boundary element tearing and interconnecting (BETI) method (a boundary element counterpart of the FETI method) has been developed by Steinbach et al. for strongly elliptic problems $[28,30,36]$. Its extension to Maxwell's equations was pursued by Windisch in his Ph.D. thesis [39], Chapter 8, but effective preconditioning for this formulation remains open. 
An indirect "single-trace" boundary integral formulation for (1.1) was proposed in [29] along with the claim that it was amenable to Calderón preconditioning. Some numerical evidence is given, but no rigorous analysis of this formulation is available.

This article is devoted to the rigorous mathematical analysis of the new multi-trace boundary integral formulation and its Galerkin discretization by means of surface edge elements. Big parts of it run parallel to the developments in the companion paper [19]. However, extra difficulties arise due to the use of electromagnetic trace spaces and the lack of coercivity of the electric field integral operator. Only fairly recently mathematical tools for dealing with these difficulties were devised, see [13,15,27], and, most relevant for our investigations, [6]. We successfully apply them to the new multi-trace formulation and establish asymptotic quasi-optimality of Galerkin solutions along with efficacy of our Calderón preconditioner. Thus, we hope to convince the reader that the techniques presented in [19] are relevant beyond acoustic scattering. We shall also tackle the case where the domain of propagation contains metallic parts or screens in a forthcoming article.

The mathematical analysis we are aiming for rests on rather technical foundations presented in the next three sections. In the second section, we introduce a proper functional setting for the study of Maxwell's equations, both for fields in the domain of propagation and their traces at boundaries, and we state precisely the Maxwell transmission problem that we wish to consider. In Section 3, we introduce and describe trace spaces well adapted to the multi-subdomain geometry of the problem under consideration. In Section 4 we recall some well established results concerning the representation of solutions to homogeneous Maxwell's equations by means of potential operators.

In Section 5 we provide a brief review of the single-trace (PMCHWT) formulation and then explain the crucial gap idea. In Section 6 we continue with technical investigations of the spaces of Cauchy data i.e. the traces of solutions to the homogeneous Maxwell's equations. In Section 7 and 8, we introduce new trace spaces, and use them to derive our new formulation. This step of the analysis is nearly identical to its counterpart given in [19], Sections 7-9, hence we will not give too much details. In Section 9, we prove that our new formulation satisfies a generalized Gårding identity, which is the main challenge of the present document. In Section 10, we conclude by showing that our formulation fits the framework introduced in [6] which allows to prove quasioptimal convergence of Galerkin discretizations that would satisfy certain properties. Finally, in Section 11, we point out how Calderón preconditioning can be done for the discrete multi-trace equations.

\section{Setting of the PRoblem}

We consider a partition $\mathbb{R}^{3}=\cup_{i=0}^{n} \bar{\Omega}_{i}$ where $\cup_{i=1}^{n} \bar{\Omega}_{i}$ is bounded and each $\Omega_{i}$ is a connected Lipschitz domain i.e. $\partial \Omega_{i}$ is locally the graph of a Lipschitz function (see Def. 3.28 in [31]). We also set $\Gamma:=\cup_{i=1}^{n} \partial \Omega_{i}$. Note that there may exist points where three or more subdomains are adjacent, which is precisely the situation that we wish to tackle. For each $j$ the vector $\mathbf{n}_{j}$ refers to the normal vector on $\partial \Omega_{j}$ directed toward the exterior of $\Omega_{j}$, see Figure 1.

\subsection{Function spaces}

We first introduce natural functional spaces adapted to domain based time-harmonic Maxwell equations, cf. [10], Section 2. First of all let us denote $\boldsymbol{H}^{s}(\Omega)=H^{s}(\Omega)^{3}$ for any domain $\Omega \subset \mathbb{R}^{3}$ and any $s>0$. Let curl $^{2}$ refer to the operator curl curl. For any open subset $\Omega \subset \mathbb{R}^{3}$, and define

$$
\begin{aligned}
\boldsymbol{H}(\operatorname{curl}, \Omega) & :=\left\{\mathbf{V} \in \boldsymbol{L}^{2}(\Omega) \mid\|\mathbf{V}\|_{\boldsymbol{H}(\mathbf{\operatorname { c u r l }}, \Omega)}^{2}:=\|\mathbf{V}\|_{\boldsymbol{L}^{2}(\Omega)}^{2}+\|\operatorname{curl} \mathbf{V}\|_{\boldsymbol{L}^{2}(\Omega)}^{2}<\infty\right\}, \\
\boldsymbol{H}\left(\mathbf{c u r l}^{2}, \Omega\right) & :=\left\{\mathbf{V} \in \boldsymbol{L}^{2}(\Omega) \mid\|\mathbf{V}\|_{\boldsymbol{H}\left(\mathbf{c u r l}^{2}, \Omega\right)}^{2}:=\|\mathbf{V}\|_{\boldsymbol{H}(\mathbf{c u r l}, \Omega)}^{2}+\left\|\operatorname{curl}^{2} \mathbf{V}\right\|_{\boldsymbol{L}^{2}(\Omega)}^{2}<\infty\right\} .
\end{aligned}
$$

If $\boldsymbol{H}(\Omega)$ is any one of these spaces, let $\boldsymbol{H}_{\text {loc }}(\Omega)$ refer to the set of $\mathbf{V} \in \boldsymbol{L}_{\text {loc }}^{2}(\Omega)$ such that $\varphi \mathbf{V} \in \boldsymbol{H}(\Omega)$ for all compactly supported $\varphi \in C^{\infty}\left(\mathbb{R}^{3}\right)$, and let $\boldsymbol{H}_{\text {comp }}(\bar{\Omega})$ refer to the set of $\mathbf{V} \in \boldsymbol{H}(\Omega)$ whose support is bounded. From the definition of curl in the sense of distributions we infer the following gluing condition for $\boldsymbol{H}_{\text {loc }}\left(\operatorname{curl}, \mathbb{R}^{3}\right)$. 
Lemma 2.1. For any $\mathbf{u} \in \boldsymbol{L}_{\text {loc }}^{2}\left(\mathbb{R}^{3}\right)$ such that $\left.\mathbf{u}\right|_{\Omega_{j}} \in \boldsymbol{H}_{\text {loc }}\left(\operatorname{curl}, \bar{\Omega}_{j}\right), \forall j=0 \ldots n$, we have $\mathbf{u} \in \boldsymbol{H}_{\text {loc }}\left(\operatorname{curl}, \mathbb{R}^{3}\right)$ if and only if

$$
\sum_{j=0}^{n} \int_{\Omega_{j}} \operatorname{curl}(\mathbf{u}) \cdot \mathbf{v}-\mathbf{u} \cdot \operatorname{curl}(\mathbf{v}) \mathrm{d} \mathbf{x}=0 \quad \forall \mathbf{v} \in \boldsymbol{H}_{\mathrm{comp}}\left(\operatorname{curl}, \mathbb{R}^{3}\right) .
$$

\subsection{Trace spaces and operators}

In this paragraph we briefly describe appropriate trace spaces for Maxwell's equations. For further details on this subject, we refer the reader to $[8,9,12]$.

For any Lipschitz domain $\Omega \subset \mathbb{R}^{3}$, denote $\mathbf{n}$ the unit normal vector to $\partial \Omega$ directed toward the exterior (it can be defined almost everywhere on $\partial \Omega$ ) and set $\boldsymbol{L}_{\boldsymbol{t}}^{2}(\partial \Omega)=\left\{\mathbf{V} \in L^{2}(\partial \Omega)^{3} \mid \mathbf{V} \cdot \mathbf{n}=0\right\}$ equipped with the norm of $L^{2}(\partial \Omega)^{3}$. Define the tangential trace operator $\gamma_{\mathrm{D}}: \boldsymbol{H}^{1}(\Omega) \rightarrow \boldsymbol{L}_{\boldsymbol{t}}^{2}(\partial \Omega)$ by

$$
\gamma_{\mathrm{D}}(\mathbf{U}):=\left.(\mathbf{U} \times \mathbf{n})\right|_{\partial \Omega}, \quad \mathrm{d} \forall \mathbf{U} \in \boldsymbol{H}^{1}(\Omega) .
$$

In the context of Maxwell's equations, the role of tangential traces becomes clear from the integration by parts formula

$$
\int_{\Omega} \operatorname{curl}(\mathbf{U}) \cdot \mathbf{V}-\mathbf{U} \cdot \operatorname{curl}(\mathbf{V}) \mathrm{d} \mathbf{x}=\int_{\partial \Omega}\left(\gamma_{\mathrm{D}}(\mathbf{U}) \times \gamma_{\mathrm{D}}(\mathbf{V})\right) \cdot \mathbf{n} \mathrm{d} \sigma \quad \forall \mathbf{U}, \mathbf{V} \in \boldsymbol{H}^{1}(\Omega) .
$$

The trace operator $\boldsymbol{\gamma}_{\mathrm{D}}$ continuously maps $\left(\boldsymbol{H}^{1}(\Omega),\|\|_{\boldsymbol{H}^{1}(\Omega)}\right)$ into $\left(\boldsymbol{L}^{2}(\partial \Omega),\|\|_{\boldsymbol{L}^{2}(\partial \Omega)}\right)$. We adopt the following notation for its range,

$$
\boldsymbol{H}_{\times}^{1 / 2}(\partial \Omega):=\gamma_{\mathrm{D}}\left(\boldsymbol{H}^{1}(\Omega)\right) .
$$

This trace space is strictly included into $\boldsymbol{L}_{\boldsymbol{t}}^{2}(\partial \Omega)$. We equip it with the graph norm

$$
\|\mathbf{V}\|_{\boldsymbol{H}_{\times}^{\frac{1}{2}}(\partial \Omega)}=\inf \left\{\|\mathbf{U}\|_{\boldsymbol{H}^{1}(\Omega)} \mid \mathbf{U} \in \boldsymbol{H}^{1}(\Omega) \quad \text { and } \quad \gamma_{\mathrm{D}}(\mathbf{U})=\mathbf{V}\right\},
$$

which renders it a Hilbert space and $\gamma_{\mathrm{D}}: \boldsymbol{H}^{1}(\Omega) \rightarrow \boldsymbol{H}_{\times}^{1 / 2}(\partial \Omega)$ a continuous operator. The space $\boldsymbol{H}_{\times}^{1 / 2}(\partial \Omega)$ is dense in $\boldsymbol{L}_{\boldsymbol{t}}^{2}(\partial \Omega)$, see [12], Section 2. Let us define $\boldsymbol{H}_{\times}^{-1 / 2}(\partial \Omega)$ as the topological dual to $\boldsymbol{H}_{\times}^{1 / 2}(\partial \Omega)$, and introduce the anti-symmetric pairing

$$
\langle\mathbf{u}, \mathbf{v}\rangle_{\times, \partial \Omega}=\int_{\partial \Omega}(\mathbf{u} \times \mathbf{v}) \cdot \mathbf{n} \mathrm{d} \sigma \quad \forall \mathbf{u}, \mathbf{v} \in \boldsymbol{L}_{t}^{2}(\partial \Omega) .
$$

In the sequel, the space $\boldsymbol{L}_{\boldsymbol{t}}^{2}(\partial \Omega)$ shall be embedded in $\boldsymbol{H}_{\times}^{-1 / 2}(\partial \Omega)$, by identifying any $\mathbf{u} \in \boldsymbol{L}_{\boldsymbol{t}}^{2}(\partial \Omega)$ with the continuous linear form $\mathbf{v} \mapsto\langle\mathbf{u}, \mathbf{v}\rangle_{\times, \partial \Omega}, \mathbf{v} \in \boldsymbol{H}_{\times}^{1 / 2}(\partial \Omega)$. Hence we set the notation

$$
\langle\mathbf{u}, \mathbf{v}\rangle_{\times, \partial \Omega}:=\mathbf{u}(\mathbf{v}) \quad \text { whenever } \quad \mathbf{u} \in \boldsymbol{H}_{\times}^{-1 / 2}(\partial \Omega), \quad \mathbf{v} \in \boldsymbol{H}_{\times}^{1 / 2}(\partial \Omega) .
$$

As in [12], Section 3, let $H^{3 / 2}(\partial \Omega)$ stand for the formal trace space of $H^{2}(\Omega)$. Then define $\nabla_{\partial \Omega}(v):=(\nabla p \times$ $\mathbf{n}) \times\left.\mathbf{n}\right|_{\partial \Omega}$ so that $\mathbf{n} \times \nabla_{\partial \Omega}(v)=\gamma_{\mathrm{D}}(\nabla p) \in \boldsymbol{H}_{\times}^{1 / 2}(\partial \Omega)$ for any $v \in H^{3 / 2}(\partial \Omega)$ such that $v=\left.p\right|_{\partial \Omega}$ for some $p \in H^{2}(\Omega)$. Further, for any $\mathbf{u} \in \boldsymbol{H}_{\times}^{-1 / 2}(\partial \Omega)$, define the surface divergence $\operatorname{div} \partial \Omega(\mathbf{u}) \in H^{-3 / 2}(\partial \Omega)$ as adjoint

$$
\left\langle\operatorname{div}_{\partial \Omega}(\mathbf{u}), v\right\rangle_{\partial \Omega}:=-\left\langle\mathbf{u}, \mathbf{n} \times \nabla_{\partial \Omega}(v)\right\rangle_{\times, \partial \Omega} \quad \forall \mathbf{u} \in \boldsymbol{H}_{\times}^{-1 / 2}(\partial \Omega), \quad \forall v \in H^{3 / 2}(\partial \Omega),
$$

where $\langle,\rangle_{\partial \Omega}$ refers to the duality pairing between $H^{+s}(\partial \Omega)$ and $H^{-s}(\partial \Omega)$ for any $s>0$. Let us introduce the space

$$
\boldsymbol{H}^{-\frac{1}{2}}(\operatorname{div}, \partial \Omega)=\left\{\mathbf{v} \in \boldsymbol{H}_{\times}^{-1 / 2}(\partial \Omega) \mid \operatorname{div}_{\partial \Omega}(\mathbf{v}) \in H^{-1 / 2}(\partial \Omega)\right\}
$$


equipped with the graph norm

$$
\|\mathbf{v}\|_{\boldsymbol{H}^{-1 / 2}(\operatorname{div}, \partial \Omega)}^{2}:=\|\mathbf{v}\|_{\boldsymbol{H}_{\times}^{-1 / 2}(\partial \Omega)}^{2}+\left\|\operatorname{div}_{\partial \Omega}(\mathbf{v})\right\|_{H^{-1 / 2}(\partial \Omega)}^{2} .
$$

An important result is that the pairing $\langle\cdot, \cdot\rangle_{\times, \partial \Omega}$ puts the space $\boldsymbol{H}^{-1 / 2}(\operatorname{div}, \partial \Omega)$ in self-duality, see [12], Lemma 5.6.

Theorem 2.2 (self-duality of $\left.\boldsymbol{H}^{-1 / 2}(\operatorname{div}, \partial \Omega)\right)$. The pairing $\langle\cdot, \cdot\rangle_{\times, \partial \Omega}$ can be extended to a continuous bilinear form over $\boldsymbol{H}^{-1 / 2}(\operatorname{div}, \partial \Omega) \times \boldsymbol{H}^{-1 / 2}(\operatorname{div}, \partial \Omega)$. For any $\varphi \in \boldsymbol{H}^{-1 / 2}(\operatorname{div}, \partial \Omega)^{\prime}$ there exists a unique $\mathbf{u}_{\varphi} \in \boldsymbol{H}^{-1 / 2}(\operatorname{div}, \partial \Omega)$ such that $\varphi(\mathbf{v})=\left\langle\mathbf{u}_{\varphi}, \mathbf{v}\right\rangle_{\times, \partial \Omega}$ for all $\mathbf{v} \in \boldsymbol{H}^{-1 / 2}(\operatorname{div}, \partial \Omega)$, and the map $\varphi \mapsto \mathbf{u}_{\varphi}$ is a continuous isomorphism.

Since $\boldsymbol{H}(\mathbf{c u r l}, \Omega)$ is the proper energy space for Maxwell's equations, we need an extension of $\gamma_{\mathrm{D}}$ to $\boldsymbol{H}(\mathbf{c u r l}, \Omega)$. This is provided by the following theorem, see [12], Section 4 .

Theorem 2.3 (tangential trace theorem for $\boldsymbol{H}(\operatorname{curl}, \Omega)$ ). The trace operator $\gamma_{\mathrm{D}}$ can be extended to a continuous map from $\boldsymbol{H}(\mathbf{c u r l}, \Omega)$ onto $\boldsymbol{H}^{-1 / 2}(\operatorname{div}, \partial \Omega)$. The operator $\boldsymbol{\gamma}_{\mathrm{D}}: \boldsymbol{H}(\mathbf{c u r l}, \Omega) \rightarrow \boldsymbol{H}^{-1 / 2}(\operatorname{div}, \partial \Omega)$ admits a continuous right-inverse. Besides the following integration by parts formula holds

$$
\int_{\partial \Omega} \operatorname{curl}(\mathbf{U}) \cdot \mathbf{V}-\mathbf{U} \cdot \operatorname{curl}(\mathbf{V}) \mathrm{d} \mathbf{x}=\left\langle\gamma_{\mathrm{D}}(\mathbf{U}), \gamma_{\mathrm{D}}(\mathbf{V})\right\rangle_{\times, \partial \Omega} \quad \forall \mathbf{U}, \mathbf{V} \in \boldsymbol{H}(\operatorname{curl}, \Omega) .
$$

As indicated by the subscript $\mathrm{D}$, in our analysis the operator $\gamma_{\mathrm{D}}$ will play a role analogous to that of the Dirichlet trace for the scalar wave scattering problem. We also introduce a counterpart for the Neumann trace: define $\boldsymbol{\gamma}_{\mathrm{N}}: \boldsymbol{H}\left(\mathbf{c u r l}^{2}, \Omega\right) \rightarrow \boldsymbol{H}^{-1 / 2}(\operatorname{div}, \partial \Omega)$ by

$$
\gamma_{\mathrm{N}}(\mathbf{U}):=\gamma_{\mathrm{D}}(\operatorname{curl} \mathbf{U}) \quad \forall \mathbf{U} \in \boldsymbol{H}\left(\operatorname{curl}^{2}, \Omega\right)
$$

By [10], Lemma 3, the operator $\gamma_{\mathrm{N}}$ is continuous, surjective, and admits a continuous right-inverse. Note that Definition (2.3) does not seem to correspond to a usual notation, see for example [10,13], where the wave number is incorporated into the definition of the trace operator. We prefer (2.3), because it will mean substantial simplifications in many computations.

\subsection{Traces local to each subdomain}

Recall that $\mathbf{n}_{j}$ refers to the normal vector to $\partial \Omega_{j}$ directed toward the exterior of $\Omega_{j}$. In the sequel we shall denote by $\gamma_{\mathrm{D}}^{j}$ and $\gamma_{\mathrm{N}}^{j}$ the interior trace with respect to $\Omega_{j}$. More precisely,

$$
\boldsymbol{\gamma}_{\mathrm{D}}^{j}(\mathbf{U}):=\left.\left(\left.\mathbf{U}\right|_{\Omega_{j}} \times \mathbf{n}_{j}\right)\right|_{\partial \Omega_{j}} \quad \text { and } \quad \boldsymbol{\gamma}_{\mathrm{N}}^{j}(\mathbf{U}):=\boldsymbol{\gamma}_{\mathrm{D}}^{j}\left(\left.\operatorname{curl} \mathbf{U}\right|_{\Omega_{j}}\right) \quad \forall \mathbf{U} \in \boldsymbol{H}_{\mathrm{loc}}\left(\operatorname{curl}^{2}, \Omega_{j}\right) .
$$

Besides, $\gamma_{\mathrm{D}, c}^{j}, \gamma_{\mathrm{N}, c}^{j}$ will refer to the same trace operators but taken from the exterior, still based on a normal vector directed toward the exterior of $\Omega_{j}$. Finally we will need averages and jumps of combinations of these traces

$$
\begin{aligned}
\gamma^{j}(\mathbf{U}) & :=\left(\gamma_{\mathrm{D}}^{j}(\mathbf{U}), \gamma_{\mathrm{N}}^{j}(\mathbf{U})\right)^{\top} \text { and } \gamma_{c}^{j}(\mathbf{U}):=\left(\gamma_{\mathrm{D}, c}^{j}(\mathbf{U}), \gamma_{\mathrm{N}, c}^{j}(\mathbf{U})\right)^{\top} \\
{\left[\gamma^{j}\right] } & :=\gamma^{j}-\gamma_{c}^{j} \quad \text { and } \quad\left\{\boldsymbol{\gamma}^{j}\right\}:=\frac{1}{2}\left(\gamma^{j}+\gamma_{c}^{j}\right) .
\end{aligned}
$$




\subsection{Transmission conditions}

In the present article, we wish to study the scattering of an electromagnetic wave by an object composed of subdomains, each of which corresponds to a homogeneous medium with particular material properties, expressed through the two coefficients

$$
\epsilon_{j} \text { (permittivity), } \mu_{j} \text { (permeability) } \in(0,+\infty) \quad j=0 \ldots n .
$$

In the transmission problem that we consider, the coefficients $\mu_{j}$ come into play through transmission conditions imposed at the interface between two subdomains $\partial \Omega_{j} \cap \partial \Omega_{k}, j, k=0 \ldots n$. These transmission conditions are usually stated as

$$
\forall j, k=0 \ldots n\left\{\begin{array}{l}
\gamma_{\mathrm{D}}^{j}(\mathbf{U})+\gamma_{\mathrm{D}}^{k}(\mathbf{U})=\mathbf{0} \\
\mu_{j}^{-1} \boldsymbol{\gamma}_{\mathrm{N}}^{j}(\mathbf{U})+\mu_{k}^{-1} \boldsymbol{\gamma}_{\mathrm{N}}^{k}(\mathbf{U})=\mathbf{0}
\end{array} \quad \text { on } \partial \Omega_{j} \cap \partial \Omega_{k},\right.
$$

for local solutions $\mathbf{U} \in \boldsymbol{H}\left(\operatorname{curl}^{2}, \bar{\Omega}_{j}\right), j=0 \ldots n$, which we would like to compute. Although (2.4) is meaningful in the sense of distributions, it is not clear whether the restriction to $\partial \Omega_{j} \cap \partial \Omega_{k}$ is a continuous operation in the trace spaces $\boldsymbol{H}^{-1 / 2}\left(\operatorname{div}, \partial \Omega_{j}\right)$ and $\boldsymbol{H}^{-1 / 2}\left(\operatorname{div}, \partial \Omega_{k}\right)$. Hence it is not clear whether (2.4) fits the setting of trace spaces introduced in the previous section. This is the reason why we choose to write the transmission conditions in a different manner: consider the function $\mu: \mathbb{R}^{3} \rightarrow(0,+\infty)$ defined by

$$
\mu(\mathbf{x})=\mu_{j} \text { in } \Omega_{j} \quad \text { with } \mu_{j} \in(0,+\infty) \forall j=0 \ldots n .
$$

Then we rewrite conditions (2.4) in the more compact form

$$
\mathbf{U} \in \boldsymbol{H}_{\mathrm{loc}}\left(\operatorname{curl}, \mathbb{R}^{3}\right) \quad \text { and } \quad \mu^{-1} \operatorname{curl} \mathbf{U} \in \boldsymbol{H}_{\mathrm{loc}}\left(\operatorname{curl}, \mathbb{R}^{3}\right) .
$$

It is straightforward to check, by means of integration by parts (2.2), that such conditions are equivalent to (2.4) in the sense of distributions.

\subsection{Electromagnetic scattering problem}

Let $\omega>0$ refer to the (angular) frequency of the electromagnetic field. Denote by $\kappa_{j}:=\omega \sqrt{\mu_{j} \epsilon_{j}}$ the wavenumber in each subdomain $\Omega_{j}$. Let $\left(\boldsymbol{e}_{\text {inc }}, \boldsymbol{h}_{\text {inc }}\right) \in \boldsymbol{H}_{\text {loc }}\left(\operatorname{curl}^{2}, \mathbb{R}^{3}\right)^{2}$ be some incident field i.e. curl $\boldsymbol{e}_{\text {inc }}-\boldsymbol{\imath} \omega \mu_{0} \boldsymbol{h}_{\text {inc }}=\mathbf{0}$ in $\mathbb{R}^{3}$, and $\operatorname{curl} \boldsymbol{h}_{\mathrm{inc}}+\boldsymbol{\imath} \omega \epsilon_{0} \boldsymbol{e}_{\mathrm{inc}}=\mathbf{0}$ in $\mathbb{R}^{3}$. Let $\boldsymbol{e}, \boldsymbol{h}$ refer to the total electric and magnetic fields, and suppose that they satisfy Maxwell's equations in frequency domain with Silver-Müller radiation conditions [20], Section 6.1

$$
\left\{\begin{array}{l}
(\boldsymbol{e}, \boldsymbol{h}) \in \boldsymbol{H}_{\mathrm{loc}}\left(\operatorname{curl}, \mathbb{R}^{3}\right)^{2} \quad \text { such that } \\
\operatorname{curl} \boldsymbol{e}-\boldsymbol{\imath} \omega \mu \boldsymbol{h}=\mathbf{0} \quad \text { in } \Omega_{j}, \text { and } \\
\operatorname{curl} \boldsymbol{h}+\boldsymbol{\imath} \omega \epsilon \boldsymbol{e}=\mathbf{0} \quad \text { in } \Omega_{j}, j=0 \ldots n, \\
\lim _{r \rightarrow+\infty} \int_{\partial \mathcal{B}_{r}}\left|\left(\boldsymbol{h}-\boldsymbol{h}_{\mathrm{inc}}\right) \times \mathbf{n}_{r}-\boldsymbol{\imath} \kappa_{0}\left(\boldsymbol{e}-\boldsymbol{e}_{\mathrm{inc}}\right)\right|^{2} \mathrm{~d} \sigma_{r}=0,
\end{array}\right.
$$

where $\mathcal{B}_{r}$ is the ball around $\mathbf{0}$ with radius $r$, and $\mathbf{n}_{r}$ is the unit vector normal to $\partial \mathcal{B}_{r}$ directed toward the exterior of $\mathcal{B}_{r}$. Observe that the first two equations in (2.6) contain the transmission conditions (2.5), since they imply that $\boldsymbol{e} \in \boldsymbol{H}_{\text {loc }}\left(\operatorname{curl}, \mathbb{R}^{3}\right)$ and $\mu^{-1} \operatorname{curl} \boldsymbol{e} \in \boldsymbol{H}_{\text {loc }}\left(\operatorname{curl}, \mathbb{R}^{3}\right)$. Renaming $\mathbf{u}=\boldsymbol{e}$ and $\mathbf{u}_{\text {inc }}=\boldsymbol{e}_{\mathrm{inc}}$, we can also 
rewrite (2.6) as a 2nd-order equation:

$$
\left\{\begin{array}{l}
\text { Find } \mathbf{u} \in \boldsymbol{H}_{\mathrm{loc}}\left(\operatorname{curl}, \bar{\Omega}_{j}\right) \quad \text { such that } \\
\operatorname{curl}(\operatorname{curl} \mathbf{u})-\kappa_{j}^{2} \mathbf{u}=\mathbf{0} \quad \text { in } \Omega_{j}, \quad j=0 \ldots n \\
\lim _{r \rightarrow+\infty} \int_{\partial \mathcal{B}_{r}}\left|\operatorname{curl}\left(\mathbf{u}-\mathbf{u}_{\mathrm{inc}}\right) \times \mathbf{n}_{r}-\boldsymbol{\imath} \kappa_{0}\left(\mathbf{u}-\mathbf{u}_{\mathrm{inc}}\right)\right|^{2} \mathrm{~d} \sigma_{r}=0 \\
\mathbf{u} \in \boldsymbol{H}_{\mathrm{loc}}\left(\operatorname{curl}, \mathbb{R}^{3}\right) \quad \text { and } \quad \mu^{-1} \mathbf{c u r l} \mathbf{u} \in \boldsymbol{H}_{\mathrm{loc}}\left(\operatorname{curl}, \mathbb{R}^{3}\right) .
\end{array}\right.
$$

Problem (2.7) is well posed, see for example [6], Theorem 4.7.

\subsubsection{Remark on the dissipative case}

We may consider a more general situation where $\epsilon_{j} \neq 0$ with $\Im m\left\{\epsilon_{j}\right\} \geq 0$ and $\Re e\left\{\epsilon_{j}\right\} \geq 0, \forall j=0 \ldots n$ (where the radiation condition would be modified accordingly). Well posedness of (2.7) in this case can be obtained by adapting [38], Section 2, and using [23], Section 5.1. In this context, as problem (2.7) remains well posed, only minor changes would be required in our analysis, $c f$. [19], and all the results presented in the sequel could be adapted. In particular Propositions 6.1, 8.2, Theorem 9.6 still hold in this case.

\section{TRACE SPACES ADAPted TO MULTI-SUbDOMAin GEOMETRIES}

In this section we introduce trace function spaces, built upon the setting described in Section 2.2, that are well adapted to integral equation formulations of problem (2.7). These spaces are a vectorial counterparts of the spaces considered in [19], Section 2. Our choices take the cue from the work of Bendali and co-workers on classical single-trace formulation of Maxwell's equations for diffraction by composite structures $[4,5]$.

\subsection{Multi-trace space}

Since we wish to derive an integral formulation for (2.7), the cartesian product of trace spaces of all subdomains appears as a simple and natural setting. Let us define the combined trace space

$$
\begin{aligned}
& \mathbb{H}(\Gamma):=\prod_{j=0}^{n} \mathbb{H}\left(\partial \Omega_{j}\right) \quad \text { where } \quad \mathbb{H}\left(\partial \Omega_{j}\right)=\boldsymbol{H}^{-\frac{1}{2}}\left(\operatorname{div}, \partial \Omega_{j}\right) \times \boldsymbol{H}^{-\frac{1}{2}}\left(\operatorname{div}, \partial \Omega_{j}\right) \\
& \text { with } \quad\|\mathbf{U}\|_{\mathbb{H}}=\left(\sum_{j=0}^{n}\left\|\mathbf{u}_{j}\right\|_{\boldsymbol{H}^{-1 / 2}\left(\operatorname{div}, \partial \Omega_{j}\right)}^{2}+\left\|\mathbf{p}_{j}\right\|_{\boldsymbol{H}^{-1 / 2}\left(\operatorname{div}, \partial \Omega_{j}\right)}^{2}\right)^{\frac{1}{2}} \text { if } \mathbf{U}=\left(\begin{array}{c}
\mathbf{u}_{j} \\
\mathbf{p}_{j}
\end{array}\right)_{j=0 \ldots n} .
\end{aligned}
$$

Equipped with such a norm, $\mathbb{H}(\Gamma)$ is a Hilbert space. In this space, we shall consider the following skew symmetric duality pairing

$$
\begin{aligned}
& \mathrm{B}(\mathbf{U}, \mathbf{V})=\sum_{j=0}^{n} \mathrm{~B}_{j}\left(\mathbf{U}_{j}, \mathbf{V}_{j}\right) \quad \text { where } \quad \mathrm{B}_{j}\left(\mathbf{U}_{j}, \mathbf{V}_{j}\right)=\left\langle\mathbf{u}_{j}, \mathbf{q}_{j}\right\rangle_{\times, \partial \Omega_{j}}-\left\langle\mathbf{v}_{j}, \mathbf{p}_{j}\right\rangle_{\times, \partial \Omega_{j}} \\
& \text { for any } \quad \mathbf{U}=\left(\begin{array}{c}
\mathbf{u}_{j} \\
\mathbf{p}_{j}
\end{array}\right)_{j=0 \ldots n} \in \mathbb{H}(\Gamma), \quad \mathbf{V}=\left(\begin{array}{c}
\mathbf{v}_{j} \\
\mathbf{q}_{j}
\end{array}\right)_{j=0 \ldots n} \in \mathbb{H}(\Gamma) .
\end{aligned}
$$

There are many possible choices for a duality pairing on $\mathbb{H}(\Gamma)$, but (3.1) will be particularly convenient for the forthcoming analysis. This pairing is non-degenerate: $\mathbf{U}=\mathbf{0} \Longleftrightarrow \mathrm{B}(\mathbf{U}, \mathbf{V})=\mathbf{0}, \forall \mathbf{V} \in \mathbb{H}(\Gamma)$. 


\subsection{Single-trace space}

As in [19], we introduce trace spaces adapted to transmission conditions, whose definition does not rely on any orientation of the interfaces $\partial \Omega_{k} \cap \partial \Omega_{j}$. We set

$$
\begin{aligned}
& \boldsymbol{X}(\Gamma):=\left\{\left(\mathbf{v}_{j}\right) \in \prod_{j=0}^{n} \boldsymbol{H}^{-\frac{1}{2}}\left(\operatorname{div}, \partial \Omega_{j}\right) \mid \exists \mathbf{V} \in \boldsymbol{H}_{\mathrm{loc}}\left(\operatorname{curl}, \mathbb{R}^{3}\right) \text { with } \mathbf{v}_{j}=\boldsymbol{\gamma}_{\mathrm{D}}^{j}(\mathbf{V})\right\} \\
& \mathbb{X}(\Gamma):=\left\{\left(\begin{array}{c}
\mathbf{v}_{j} \\
\mathbf{q}_{j}
\end{array}\right) \in \mathbb{H}(\Gamma) \mid\left(\mathbf{v}_{j}\right),\left(\mathbf{q}_{j}\right) \in \boldsymbol{X}(\Gamma)\right\} .
\end{aligned}
$$

The single-trace space $\mathbb{X}(\Gamma)$ is closed in $\mathbb{H}(\Gamma)$ for \|\|$_{\mathbb{H}}$, as it is defined by constraints involving continuous functionals. Here is yet another instructive remark; for any $j=0 \ldots n$, assume that $\mathbf{v} \in \boldsymbol{H}_{\text {loc }}\left(\mathbf{c u r l}^{2}, \mathbb{R}^{3} \backslash \Omega_{j}\right)$, and consider $\mathbf{V}=\left(\mathbf{V}_{q}\right)_{q=0 \ldots n}$ where $\mathbf{V}_{q}=\gamma^{q}(\mathbf{v})$ if $q \neq j$ and $\mathbf{V}_{j}=\gamma_{c}^{j}(\mathbf{v})$. Then $\mathbf{V} \in \mathbb{X}(\Gamma)$. The following result provides yet another characterization of $\mathbb{X}(\Gamma)$, which amounts to a weak version of the transmission conditions $(2.5)$.

Proposition 3.1. For any $\mathbf{U} \in \mathbb{H}(\Gamma)$ we have: $\mathbf{U} \in \mathbb{X}(\Gamma) \Longleftrightarrow \mathrm{B}(\mathbf{U}, \mathbf{V})=0, \forall \mathbf{V} \in \mathbb{X}(\Gamma)$

Proof. The proof is very similar to the proof of Proposition 2.1 in [18] and is elementary, but we reproduce it here so that the reader can gain some familiarity with the space $\mathbb{X}(\Gamma)$. According to the definition of $\mathrm{B}($,$) , it$ suffices to show that for $\left(\mathbf{v}_{j}\right) \in \Pi_{j=0}^{n} \boldsymbol{H}^{-1 / 2}\left(\operatorname{div}, \partial \Omega_{j}\right)$ we have

$$
\left(\mathbf{v}_{j}\right) \in \boldsymbol{X}(\Gamma) \Longleftrightarrow \sum_{j=0}^{n}\left\langle\mathbf{v}_{j}, \mathbf{q}_{j}\right\rangle_{\times, \partial \Omega_{j}}=0 \quad \forall\left(\mathbf{q}_{j}\right) \in \boldsymbol{X}(\Gamma) .
$$

First, assume that $\left(\mathbf{v}_{j}\right) \in \boldsymbol{X}(\Gamma)$, and take some $\mathbf{v} \in \boldsymbol{H}\left(\mathbf{c u r l}, \mathbb{R}^{3}\right)$ such that $\boldsymbol{\gamma}_{\mathrm{D}}^{j}(\mathbf{v})=\mathbf{v}_{j} \forall j=0 \ldots n$. Consider an arbitrary $\left(\mathbf{q}_{j}\right) \in \boldsymbol{X}(\Gamma)$ and some $\mathbf{q} \in \boldsymbol{H}\left(\mathbf{c u r l}, \mathbb{R}^{3}\right)$ with compact support such that $\boldsymbol{\gamma}^{j}(\mathbf{q})=\mathbf{q}_{j} \forall j=0 \ldots n$. According to (2.2) and Lemma 2.1, we have

$$
\sum_{j=0}^{n}\left\langle\mathbf{v}_{j}, \mathbf{q}_{j}\right\rangle_{\times, \partial \Omega_{j}}=\sum_{j=0}^{n} \int_{\Omega_{j}} \operatorname{curl}(\mathbf{v}) \cdot \mathbf{q}-\mathbf{v} \cdot \operatorname{curl}(\mathbf{q}) \mathrm{d} \mathbf{x}=0 .
$$

Now assume that $\left(\mathbf{v}_{j}\right) \in \Pi_{j=0}^{n} \boldsymbol{H}^{-1 / 2}\left(\operatorname{div}, \partial \Omega_{j}\right)$ only satisfies the condition on the right hand side in (3.2). Take $\mathbf{u} \in \boldsymbol{L}^{2}\left(\mathbb{R}^{3}\right)$ such that $\left.\mathbf{u}\right|_{\Omega_{j}} \in \boldsymbol{H}\left(\operatorname{curl}, \bar{\Omega}_{j}\right)$ and $\boldsymbol{\gamma}_{\mathrm{D}}^{j}(\mathbf{u})=\mathbf{v}_{j}, \forall j=0 \ldots$. Since $\left(\boldsymbol{\gamma}_{\mathrm{D}}^{j}(\mathbf{q})\right) \in \boldsymbol{X}(\Gamma)$ whenever $\mathbf{q} \in \boldsymbol{H}_{\text {comp }}\left(\right.$ curl, $\left.\mathbb{R}^{3}\right)$ we have

$$
\sum_{j=0}^{n} \int_{\Omega_{j}} \operatorname{curl}(\mathbf{u}) \cdot \mathbf{q}-\mathbf{u} \cdot \operatorname{curl}(\mathbf{q}) \mathrm{d} \mathbf{x}=\sum_{j=0}^{n}\left\langle\mathbf{v}_{j}, \gamma_{\mathrm{D}}^{j}(\mathbf{q})\right\rangle_{\times, \partial \Omega_{j}}=0 \quad \forall \mathbf{q} \in \boldsymbol{H}_{\mathrm{comp}}\left(\operatorname{curl}, \mathbb{R}^{3}\right) .
$$

This implies that $\mathbf{u} \in \boldsymbol{H}_{\text {loc }}\left(\mathbf{c u r l}, \mathbb{R}^{3}\right)$ according to Lemma 2.1. Since $\mathbf{v}_{j}=\boldsymbol{\gamma}_{\mathrm{D}}^{j}(\mathbf{u})$, we conclude that $\left(\mathbf{v}_{j}\right) \in \boldsymbol{X}(\Gamma)$.

The single-trace space $\mathbb{X}(\Gamma)$ is particularly convenient for dealing with transmission conditions. Indeed, according to the discussion of Section 2.4, for any vector field $\mathbf{u} \in \boldsymbol{L}_{\mathrm{loc}}^{2}\left(\mathbb{R}^{3}\right)$ such that $\left.\mathbf{u}\right|_{\Omega_{j}} \in \boldsymbol{H}_{\mathrm{loc}}\left(\operatorname{curl}^{2}, \bar{\Omega}_{j}\right)$ we have

$$
\text { u satisfies }(2.4) \quad \Longleftrightarrow \quad\left(\mathcal{T}_{\mu_{j}} \gamma^{j}(\mathbf{u})\right)_{j=0 \ldots n} \in \mathbb{X}(\Gamma) \quad \text { where } \quad \mathcal{T}_{\mu}:=\left[\begin{array}{ll}
1 & 0 \\
0 & 1 / \mu
\end{array}\right] .
$$

We shall also consider the scaling operator $\mathrm{T}_{\mu}: \mathbb{H}(\Gamma) \rightarrow \mathbb{H}(\Gamma)$ defined by $\mathrm{T}_{\mu}(U):=\left(\mathcal{T}_{\mu_{0}}\left(U_{0}\right), \ldots, \mathcal{T}_{\mu_{n}}\left(U_{n}\right)\right)$ for any $U=\left(U_{0}, \ldots, U_{n}\right) \in \mathbb{H}(\Gamma)$. Finally we define $\mathrm{T}_{0}(U)=\left(\mathcal{T}_{\mu_{0}}\left(U_{0}\right), \ldots, \mathcal{T}_{\mu_{0}}\left(U_{n}\right)\right)$. In particular, we have the following property $\mathrm{T}_{0}(\mathbb{X}(\Gamma))=\mathbb{X}(\Gamma)$. 


\section{Potentials}

In this section, we recall already well established results concerning potential operators and representation results for Maxwell's equations. These results were reported in detail in [10] and were proved in $[11,22,24]$.

\subsection{Representation formula}

Let $\mathscr{G}_{\kappa}(\mathbf{x})=\exp (\boldsymbol{\imath} \kappa|\mathbf{x}|) /(4 \pi|\mathbf{x}|)$ refer to the radiating fundamental solution for the operator $-\Delta-\kappa^{2}$. First, for any subdomain $\Omega_{j}$, we introduce the intermediate potentials

$$
\begin{aligned}
& \Psi_{\kappa}^{j}(q)(\mathbf{x})=\int_{\partial \Omega_{j}} \mathscr{G}_{\kappa}(\mathbf{x}-\mathbf{y}) q(\mathbf{y}) \mathrm{d} \sigma(\mathbf{y}) \quad \forall q \in H^{-\frac{1}{2}}\left(\partial \Omega_{j}\right), \\
& \boldsymbol{\Psi}_{\kappa}^{j}(\mathbf{p})(\mathbf{x})=\int_{\partial \Omega_{j}} \mathscr{G}_{\kappa}(\mathbf{x}-\mathbf{y}) \mathbf{p}(\mathbf{y}) \mathrm{d} \sigma(\mathbf{y}) \quad \forall \mathbf{p} \in \boldsymbol{H}_{\times}^{-\frac{1}{2}}\left(\partial \Omega_{j}\right) .
\end{aligned}
$$

According to $[11,22]$, these potentials give rise to continuous mappings $\Psi_{\kappa}^{j}: H^{-1 / 2}\left(\partial \Omega_{j}\right) \rightarrow H_{\mathrm{loc}}^{1}\left(\mathbb{R}^{3}\right)$ and $\boldsymbol{\Psi}_{\kappa}^{j}: \boldsymbol{H}_{\times}^{-1 / 2}\left(\partial \Omega_{j}\right) \rightarrow \boldsymbol{H}_{\text {loc }}^{1}\left(\mathbb{R}^{3}\right)$. Based on them, we introduce the electromagnetic counterparts of the single and double layer potentials, $c f$. [10], Section 4 :

$$
\begin{aligned}
\mathbf{S L}_{\kappa}^{j}(\mathbf{q})(\mathbf{x}) & :=\Psi_{\kappa}^{j}(\mathbf{q})(\mathbf{x})+\kappa^{-2} \nabla\left(\Psi_{\kappa}^{j}\left(\operatorname{div}_{\partial \Omega_{j}}(\mathbf{q})\right)\right)(\mathbf{x}), & \\
\mathbf{D L}_{\kappa}^{j}(\mathbf{v})(\mathbf{x}) & :=\operatorname{curl}\left(\Psi_{\kappa}^{j}(\mathbf{v})\right)(\mathbf{x}), & \mathbf{v}, \mathbf{q} \in \boldsymbol{H}^{-\frac{1}{2}}\left(\operatorname{div}, \partial \Omega_{j}\right) . \\
\mathbf{G}_{\kappa}^{j}\left(\left[\begin{array}{l}
\mathbf{v} \\
\mathbf{q}
\end{array}\right]\right)(\mathbf{x}) & :=\mathbf{D L}_{\kappa}^{j}(\mathbf{v})(\mathbf{x})+\mathbf{S L}_{\kappa}^{j}(\mathbf{q})(\mathbf{x}), &
\end{aligned}
$$

For any $\mathbf{v}, \mathbf{q} \in \boldsymbol{H}^{-1 / 2}\left(\operatorname{div}, \partial \Omega_{j}\right)$ the vector fields $\mathbf{S L}_{\kappa}^{j}(\mathbf{q})(\mathbf{x})$ and $\mathbf{D} \mathbf{L}_{\kappa}^{j}(\mathbf{v})(\mathbf{x})$ are solutions to Maxwell's equations in each subdomain and satisfy the Silver-Müller radiation condition. Besides, the operator $\mathbf{G}_{\kappa}^{j}: \mathbb{H}\left(\partial \Omega_{j}\right) \rightarrow$ $\Pi_{k=0}^{n} \boldsymbol{H}_{\text {loc }}\left(\operatorname{curl}^{2}, \bar{\Omega}_{k}\right) \cap \boldsymbol{L}_{\text {loc }}^{2}\left(\mathbb{R}^{3}\right)$ is continuous for any $j=0 \ldots n$. A crucial result concerning these potential operators is that when $\mathbf{u}$ is a solution to Maxwell's equations in $\Omega_{j}$, then $\mathbf{G}_{\kappa}^{j}$ can be used to reconstruct $\mathbf{u}$ from its traces on $\partial \Omega_{j}$, see [10], Theorem 6 .

Theorem 4.1 (stratton-Chu representation formula). Let $\mathbf{u} \in \boldsymbol{H}_{\mathrm{loc}}\left(\mathbf{c u r l}^{2}, \bar{\Omega}_{j}\right)$ satisfy the Maxwell equations $\operatorname{curl}(\operatorname{curl} \mathbf{u})-\kappa_{j}^{2} \mathbf{u}=\mathbf{0}$ in $\Omega_{j}$. In the case where $j=0$, in addition assume that it satisfies the Silver-Müller radiation condition $\lim _{r \rightarrow+\infty} \int_{\partial \mathcal{B}_{r}}\left|\mathbf{c u r l} \mathbf{u} \times \mathbf{n}_{r}-\boldsymbol{\imath} \kappa_{0} \mathbf{u}\right|^{2} \mathrm{~d} \sigma_{r}=0$. Then we have

$$
\mathbf{G}_{\kappa}^{j}\left(\gamma^{j}(\mathbf{u})\right)(\mathbf{x})=\left\{\begin{array}{cl}
\mathbf{u}(\mathbf{x}) & \text { if } \mathbf{x} \in \Omega_{j} \\
\mathbf{0} & \text { if } \mathbf{x} \in \mathbb{R}^{3} \backslash \bar{\Omega}_{j} .
\end{array}\right.
$$

Similarly, if $\mathbf{u} \in \boldsymbol{H}_{\mathrm{loc}}\left(\mathbf{c u r l}^{2}, \mathbb{R}^{3} \backslash \Omega_{j}\right)$ satisfies the Maxwell equations $\operatorname{curl}(\operatorname{curl} \mathbf{u})-\kappa_{j}^{2} \mathbf{u}=\mathbf{0}$ in $\mathbb{R}^{3} \backslash \Omega_{j}$, as well as the Silver-Müller radiation condition $\lim _{r \rightarrow+\infty} \int_{\partial \mathcal{B}_{r}}\left|\mathbf{c u r l} \mathbf{u} \times \mathbf{n}_{r}-\boldsymbol{\imath} \kappa_{j} \mathbf{u}\right|^{2} \mathrm{~d} \sigma_{r}=0$ except if $j=0$, then we have

$$
\mathbf{G}_{\kappa}^{j}\left(\gamma_{c}^{j}(\mathbf{u})\right)(\mathbf{x})=\left\{\begin{array}{c}
\mathbf{0} \quad \text { if } \mathbf{x} \in \Omega_{j} \\
-\mathbf{u}(\mathbf{x}) \text { if } \mathbf{x} \in \mathbb{R}^{3} \backslash \bar{\Omega}_{j} .
\end{array}\right.
$$

Another classical and important result concerns the behavior of the potentials across the boundary of the associated subdomain, summarized in the jump relations, see [10], Theorem 7 ,

$$
\left[\gamma^{j}\right] \cdot \mathbf{G}_{\kappa_{j}}^{j}(\mathbf{V})=\mathbf{V} \quad \forall \mathbf{V} \in \mathbb{H}\left(\partial \Omega_{j}\right)
$$

In the sequel we will also need a remarkable property involving the potential operators as well as the elements of $\mathbb{X}(\Gamma)$. Observe that, in the following statement only a single wave number $\kappa_{0}$ is used. 
Lemma 4.2. For any $\kappa_{0} \in(0,+\infty)$ we have

$$
\sum_{j=0}^{n} \mathbf{G}_{\kappa_{0}}^{j}\left(\mathbf{U}_{j}\right)(\mathbf{x})=0 \quad \forall \mathbf{x} \in \mathbb{R}^{3}, \quad \forall \mathbf{U}=\left(\mathbf{U}_{j}\right)_{0 \leq j \leq n} \in \mathbb{X}(\Gamma) .
$$

This result corresponds to [18], Lemma 6.1. The underlying intuition is that the jumps of the individual potentials $\mathbf{G}_{\kappa_{0}}^{j}$ across interfaces cancel each other in the sum (4.3).

Proof of Lemma 4.2. Pick any $\mathbf{U}=\left(\mathbf{U}_{j}\right)_{j=0 \ldots n} \in \mathbb{X}(\Gamma)$ that will be fixed until the end of the proof. Set $\mathbf{u}(\mathbf{x})=\sum_{j=0}^{n} \mathbf{G}_{\kappa_{0}}^{j}\left(\mathbf{U}_{j}\right)(\mathbf{x})$. We have to prove that $\mathbf{u}(\mathbf{x})=0, \forall \mathbf{x} \in \mathbb{R}^{3}$.

First, we prove that $\mathbf{u} \in \boldsymbol{H}_{\text {loc }}\left(\operatorname{curl}^{2}, \mathbb{R}^{3}\right)$. For this, since $\mathbf{u} \in \boldsymbol{H}_{\text {loc }}\left(\operatorname{curl}^{2}, \bar{\Omega}_{j}\right)$ for any $j=0 \ldots n$ as is clear from its definition, it is sufficient to show that $\left(\boldsymbol{\gamma}^{j}(\mathbf{u})\right)_{j=0 \ldots n} \in \mathbb{X}(\Gamma)$. Take any $j=0 \ldots n$, and observe that $\mathbf{G}_{\kappa_{0}}^{j}\left(\mathbf{U}_{j}\right) \in \boldsymbol{H}_{\text {loc }}\left(\mathbf{c u r l}^{2}, \mathbb{R}^{3} \backslash \Omega_{j}\right)$, so that $\left(\mathbf{W}_{q}\right)_{q=0 \ldots n} \in \mathbb{X}(\Gamma)$ where $\mathbf{W}_{q}:=\gamma^{q} \cdot \mathbf{G}_{\kappa_{0}}^{j}\left(\mathbf{U}_{j}\right)$ if $q \neq j$, and $\mathbf{W}_{j}:=\gamma_{c}^{j} \cdot \mathbf{G}_{\kappa_{0}}^{j}\left(\mathbf{U}_{j}\right)$. As a consequence, according to Proposition 3.1, we have

$$
\begin{aligned}
\sum_{q=0}^{n} \mathrm{~B}_{q}\left(\boldsymbol{\gamma}^{q} \cdot \mathbf{G}_{\kappa_{0}}^{j}\left(\mathbf{U}_{j}\right), \mathbf{V}_{q}\right) & =\mathrm{B}_{j}\left(\left[\boldsymbol{\gamma}^{j}\right] \cdot \mathbf{G}_{\kappa_{0}}^{j}\left(\mathbf{U}_{j}\right), \mathbf{V}_{j}\right)+\sum_{q=0}^{n} \mathrm{~B}_{q}\left(\mathbf{W}_{q}, \mathbf{V}_{q}\right) \\
& =\mathrm{B}_{j}\left(\mathbf{U}_{j}, \mathbf{V}_{j}\right) \quad \forall \mathbf{V}=\left(\mathbf{V}_{j}\right) \in \mathbb{X}(\Gamma) .
\end{aligned}
$$

For the second equality above, we used the fact that $\left[\gamma^{j}\right] \cdot \mathbf{G}_{\kappa_{0}}^{j}\left(\mathbf{U}_{j}\right)=\mathbf{U}_{j}$ according to (4.2). Summing all such identities over $j=0 \ldots n$, we obtain

$$
\begin{aligned}
\sum_{q=0}^{n} \mathrm{~B}_{q}\left(\boldsymbol{\gamma}^{q}(\mathbf{u}), \mathbf{V}_{q}\right) & =\sum_{j=0}^{n} \sum_{q=0}^{n} \mathrm{~B}_{q}\left(\boldsymbol{\gamma}^{q} \cdot \mathbf{G}_{\kappa_{0}}^{j}\left(\mathbf{U}_{j}\right), \mathbf{V}_{q}\right) \\
& =\sum_{j=0}^{n} \mathrm{~B}_{j}\left(\mathbf{U}_{j}, \mathbf{V}_{j}\right)=0 \quad \forall \mathbf{V}=\left(\mathbf{V}_{j}\right) \in \mathbb{X}(\Gamma)
\end{aligned}
$$

The previous identity implies that $\left(\gamma^{j}(\mathbf{u})\right) \in \mathbb{X}(\Gamma)$ according to the characterization of $\mathbb{X}(\Gamma)$ provided by Proposition 3.1, which proves that $\mathbf{u} \in \boldsymbol{H}_{\mathrm{loc}}\left(\mathbf{c u r l}^{2}, \mathbb{R}^{3}\right)$.

Now observe that $\mathbf{u}(\mathbf{x})$ is an outgoing solution to homogeneous Maxwell's equations associated to the wave number $\kappa_{0}$ in each subdomain $\Omega_{j}$, since each $\mathbf{G}_{\kappa_{0}}^{j}\left(\mathbf{U}_{j}\right)$ satisfies such equations. Besides $\mathbf{u}(\mathbf{x})$ satisfies transmission conditions since $\left(\gamma^{j}(\mathbf{u})\right) \in \mathbb{X}(\Gamma)$. As a consequence, since problem (2.7) is well posed, this implies $\mathbf{u}=0$.

\subsection{Cauchy data and Calderón projectors}

Now we introduce special trace spaces that will play an important role in the sequel. We exhibit additional properties of these spaces in the next section.

Definition 4.3. The set of interior Cauchy data associated to the subdomain $\Omega_{j}$ with respect to the wave number $\kappa_{j}$ is defined by

$$
\begin{aligned}
\mathcal{C}_{\kappa_{j}}\left(\partial \Omega_{j}\right):=\left\{\gamma^{j}(\mathbf{u}) \mid \mathbf{u} \in \boldsymbol{H}_{\mathrm{loc}}\left(\operatorname{curl}^{2}, \bar{\Omega}_{j}\right), \operatorname{curl}(\operatorname{curl} \mathbf{u})-\kappa_{j}^{2} \mathbf{u}=\mathbf{0} \text { in } \Omega_{j}\right. \\
\left.\quad \text { and } \lim _{r \rightarrow+\infty} \int_{\partial \mathcal{B}_{r}}\left|\operatorname{curl} \mathbf{u} \times \mathbf{n}_{r}-\boldsymbol{\imath} \kappa_{0} \mathbf{u}\right|^{2} \mathrm{~d} \sigma_{r}=0, \text { if } j=0\right\} .
\end{aligned}
$$

The space of global interior Cauchy data associated to $\kappa=\left(\kappa_{0}, \ldots, \kappa_{n}\right)$ is defined as the cartesian product

$$
\mathcal{C}_{\kappa}(\Gamma):=\mathcal{C}_{\kappa_{0}}\left(\partial \Omega_{0}\right) \times \cdots \times \mathcal{C}_{\kappa_{n}}\left(\partial \Omega_{n}\right) .
$$


Note that we may also consider spaces of exterior Cauchy data. However, as we will refer to exterior Cauchy data only occasionally, we do not introduce special notation for such spaces. We have the following characterization, cf. [10], Theorem 8.

Proposition 4.4 (characterization of Cauchy data). For any $j=0 \ldots n$ the operator $\gamma^{j} \cdot \mathbf{G}_{\kappa_{j}}^{j}: \mathbb{H}\left(\partial \Omega_{j}\right) \rightarrow$ $\mathbb{H}\left(\partial \Omega_{j}\right)$ is a continuous projector, called the interior Calderón projector of $\Omega_{j}$, and for any $\mathbf{V} \in \mathbb{H}\left(\partial \Omega_{j}\right)$ we have

$$
\mathbf{V} \in \mathcal{C}_{\kappa_{j}}\left(\partial \Omega_{j}\right) \quad \Longleftrightarrow \quad \mathbf{V}=\gamma^{j} \cdot \mathbf{G}_{\kappa_{j}}^{j}(\mathbf{V})
$$

As a consequence, Cauchy data spaces are closed sub-spaces of $\mathbb{H}(\Gamma)$ since they can be characterized as kernels of continuous projectors. We introduce other continuous operators $\mathrm{C}_{\kappa_{j}}^{j}: \mathbb{H}\left(\partial \Omega_{j}\right) \rightarrow \mathbb{H}\left(\partial \Omega_{j}\right)$ defined by

$$
\mathrm{Id} / 2+\mathrm{C}_{\kappa_{j}}^{j}:=\gamma^{j} \cdot \mathbf{G}_{\kappa_{j}}^{j} .
$$

Thanks to the jump relations (4.2), this definition can be rewritten $\mathrm{C}_{\kappa_{j}}^{j}=\left\{\boldsymbol{\gamma}^{j}\right\} \cdot \mathbf{G}_{\kappa_{j}}^{j}$. As a consequence of (4.4) we have $\left(2 \mathrm{C}_{\kappa_{j}}^{j}\right)^{2}=\mathrm{Id}$ and also $\mathcal{C}_{\kappa_{j}}\left(\partial \Omega_{j}\right)=\operatorname{Range}\left(\mathrm{Id} / 2+\mathrm{C}_{\kappa_{j}}^{j}\right)$. From Proposition 4.4 and Definition (4.4), we conclude

$$
\mathbf{U} \in \mathcal{C}_{\kappa}(\Gamma) \Longleftrightarrow\left(\mathrm{Id} / 2+\mathrm{C}_{\kappa}\right) \mathbf{U}=\mathbf{U} \quad \text { where } \quad \mathrm{C}_{\kappa}=\left[\begin{array}{cccc}
\mathrm{C}_{\kappa_{0}}^{0} & 0 & \cdots & 0 \\
0 & \mathrm{C}_{\kappa_{1}}^{1} & \ddots & \vdots \\
\vdots & \ddots & \ddots & 0 \\
0 & \cdots & 0 & \mathrm{C}_{\kappa_{n}}^{n}
\end{array}\right]
$$

Let us emphasize that we also have $\mathbf{V} \in \operatorname{Range}\left(\mathrm{Id} / 2-\mathrm{C}_{\kappa_{j}}^{j}\right)$ if and only if there exists some $\mathbf{v} \in \boldsymbol{H}_{\text {loc }}\left(\mathbf{c u r l}^{2}, \mathbb{R}^{3} \backslash\right.$ $\left.\Omega_{j}\right)$ such that $\gamma_{c}^{j}(\mathbf{v})=\mathbf{V}$, and such that $\operatorname{curl}(\operatorname{curl} \mathbf{v})-\kappa_{j}^{2} \mathbf{v}=\mathbf{0}$ in $\mathbb{R}^{3} \backslash \bar{\Omega}_{j}$, and $\mathbf{v}$ satisfies the Silver-Müller radiation condition, except if $j=0$.

\subsection{Scaled Calderón projectors}

Finally we introduce operators $\mathrm{A}_{\kappa_{j}, \mu_{j}}^{j}: \mathbb{H}\left(\partial \Omega_{j}\right) \rightarrow \mathbb{H}\left(\partial \Omega_{j}\right)$ and $\mathrm{A}_{\kappa, \mu}: \mathbb{H}(\Gamma) \rightarrow \mathbb{H}(\Gamma)$ such that $\mathrm{Id} / 2+$ $\mathrm{A}_{\kappa_{j}, \mu_{j}}^{j}, j=0 \ldots n$, and $\mathrm{Id} / 2+\mathrm{A}_{\kappa, \mu}$ are scaled versions of Calderón projectors well adapted to the treatment of transmission conditions. They are defined by

$$
\mathrm{A}_{\kappa_{j}, \mu_{j}}^{j}=\mathcal{T}_{\mu_{j}} \cdot \mathrm{C}_{\kappa_{j}}^{j} \cdot \mathcal{T}_{\mu_{j}}^{-1} \text { and } \mathrm{A}_{\kappa, \mu}=\mathrm{T}_{\mu} \cdot \mathrm{C}_{\kappa} \cdot \mathrm{T}_{\mu}^{-1} .
$$

It is straightforward to check that $\mathrm{Id} / 2+\mathrm{A}_{\kappa_{j}, \mu_{j}}^{j}, j=0 \ldots n$, and $\mathrm{Id} / 2+\mathrm{A}_{\kappa, \mu}$ are projectors. By analogy with the Cauchy data spaces introduced in Definition 4.3, we define

$$
\begin{aligned}
& \mathcal{C}_{\kappa_{j}, \mu_{j}}\left(\partial \Omega_{j}\right):=\text { Range }\left(\mathrm{Id} / 2+\mathrm{A}_{\kappa_{j}, \mu_{j}}^{j}\right), \\
& \mathcal{C}_{\kappa, \mu}(\Gamma):=\text { Range }\left(\mathrm{Id} / 2+\mathrm{A}_{\kappa, \mu}\right)=\mathcal{C}_{\kappa_{0}, \mu_{0}}\left(\partial \Omega_{0}\right) \times \cdots \times \mathcal{C}_{\kappa_{n}, \mu_{n}}\left(\partial \Omega_{n}\right) .
\end{aligned}
$$

We also have $\mathcal{C}_{\kappa, \mu}(\Gamma)=\operatorname{Ker}\left(-\operatorname{Id} / 2+\mathrm{A}_{\kappa, \mu}\right)$. The operator $\mathrm{A}_{\kappa, \mu}$ is symmetric with respect to the pairing $\mathrm{B}($,$) :$

\section{Lemma 4.5.}

$$
\mathrm{B}\left(\left(\mathrm{Id} / 2+\mathrm{A}_{\kappa, \mu}\right) \mathbf{U}, \mathbf{V}\right)=\mathrm{B}\left(\left(-\mathrm{Id} / 2+\mathrm{A}_{\kappa, \mu}\right) \mathbf{V}, \mathbf{U}\right) \quad \forall \mathbf{U}, \mathbf{V} \in \mathbb{H}(\Gamma) .
$$

Proof. According to [13], Theorem 3.9, we have $\mathrm{B}_{j}\left(\mathrm{C}_{\kappa_{j}}\left(\mathbf{U}_{j}\right), \mathbf{V}_{j}\right)=\mathrm{B}_{j}\left(\mathrm{C}_{\kappa_{j}}\left(\mathbf{V}_{j}\right), \mathbf{U}_{j}\right)$ for all $\mathbf{U}_{j}, \mathbf{V}_{j} \in$ $\mathbb{H}\left(\partial \Omega_{j}\right)$, and any $j=0 \ldots n$. Besides, since $\mathrm{B}_{j}\left(\mathcal{T}_{\mu_{j}}\left(\mathbf{U}_{j}\right), \mathbf{V}_{j}\right)=\mu_{j}^{-1} \mathrm{~B}_{j}\left(U_{j}, \mathcal{T}_{1 / \mu_{j}}\left(V_{j}\right)\right)$, we deduce that $\mathrm{B}_{j}\left(\mathrm{~A}_{\kappa_{j}, \mu_{j}}\left(\mathbf{U}_{j}\right), \mathbf{V}_{j}\right)=\mathrm{B}_{j}\left(\mathrm{~A}_{\kappa_{j}, \mu_{j}}\left(\mathbf{V}_{j}\right), \mathbf{U}_{j}\right)$. As $\mathrm{B}_{j}\left(\mathbf{U}_{j}, \mathbf{V}_{j}\right)=-\mathrm{B}_{j}\left(\mathbf{V}_{j}, \mathbf{U}_{j}\right)$, the result stated above is obtained by summing the previous identities over $j=0 \ldots n$. 


\section{Classical Single-trace Formulation of the First Kind}

In this section, we briefly recall the derivation of the classical PMCHWT single-trace formulation of the first kind. We adhere to a variational perspective pioneered in the work of Bendali et al. (see [4,5]) based on Rumsey's principle. It is the same perspective employed in the mathematical analysis of single-trace formulations in [38] (for acoustics) and [6] (for electromagnetics). Then, we use the single-trace formulation as a stepping stone for motivating our new multi-trace boundary integral equations via a "gap idea", cf. [19], Section 5.

In this formulation the unknown will be $\mathbf{U}=\left(\mathcal{T}_{\mu_{j}} \cdot \boldsymbol{\gamma}^{j}(\mathbf{u})\right)_{j=0 \ldots n}$ where $\mathbf{u}$ is the solution to problem (2.7). We also have to consider $\mathbf{U}^{\text {inc }}=\left(\mathcal{T}_{\mu_{0}} \cdot \gamma^{0}\left(\mathbf{u}_{\text {inc }}\right), \mathbf{0}, \ldots, \mathbf{0}\right)$. With these new notations, problem (2.7) may then be reformulated in the following manner

$$
\text { Find } \mathbf{U} \in \mathbb{X}(\Gamma) \text { such that }\left(\operatorname{Id} / 2-\mathrm{C}_{\kappa}\right) \mathrm{T}_{\mu}^{-1}\left(\mathbf{U}-\mathbf{U}^{\text {inc }}\right)=\mathbf{0} .
$$

This formulation is clearly well posed since it is exactly equivalent to problem (2.7) that is itself well posed. Let us define

$$
\mathbf{F}^{\mathrm{inc}}:=\left(\mathrm{Id} / 2-\mathrm{A}_{\kappa, \mu}\right) \mathbf{U}^{\mathrm{inc}} .
$$

With these notations, since $\mathrm{B}(\mathbf{U}, \mathbf{V})=0$ whenever $\mathbf{U}, \mathbf{V} \in \mathbb{X}(\Gamma)$, multiplying formulation (5.1) on the left by $\mathrm{T}_{\mu}$ and testing with functions $\mathbf{V} \in \mathbb{X}(\Gamma)$ we obtain

$$
\text { Find } \mathbf{U} \in \mathbb{X}(\Gamma) \text { such that } \mathrm{B}\left(\mathrm{A}_{\kappa, \mu}(\mathbf{U}), \mathbf{V}\right)=-\mathrm{B}\left(\mathbf{F}^{\text {inc }}, \mathbf{V}\right) \quad \forall \mathbf{V} \in \mathbb{X}(\Gamma) \text {. }
$$

If $\mathbf{U} \in \mathbb{X}(\Gamma)$ is solution to (5.1), then it is clearly solution to (5.2). The converse also holds, although this is much less trivial, and this implies that (5.2) is well posed. It is a consequence of the following result that was proved in [6].

Proposition 5.1 (unique solvability of single-trace formulation). Assume that $\epsilon_{j}, \mu_{j} \in(0,+\infty)$ and consider any $\mathbf{F} \in \mathbb{H}(\Gamma)$. Then there exists a unique $\mathbf{U} \in \mathbb{X}(\Gamma)$ satisfying $\mathrm{B}\left(\mathrm{A}_{\kappa, \mu}(\mathbf{U}), \mathbf{V}\right)=\mathrm{B}(\mathbf{F}, \mathbf{V}), \forall \mathbf{V} \in \mathbb{X}(\Gamma)$.

Next, let us zero in on the case of three subdomains $(n=2)$, with $\Omega_{1}$ and $\Omega_{2}$ being separated, $\partial \Omega_{1} \cap \partial \Omega_{2}=\emptyset$, which means $\Gamma=\partial \Omega_{1} \cup \partial \Omega_{2}=\partial \Omega_{0}$. In this case a simple characterization of $\mathbb{X}(\Gamma)$ is available

$$
\mathbb{X}(\Gamma)==\left\{\left(\left(\begin{array}{l}
-\mathbf{V}_{1} \\
-\mathbf{V}_{2}
\end{array}\right), \mathbf{V}_{1}, \mathbf{V}_{2}\right) \mid \mathbf{V}_{1} \in \mathbb{H}\left(\partial \Omega_{1}\right), \mathbf{V}_{2} \in \mathbb{H}\left(\partial \Omega_{2}\right)\right\},
$$

where the $\partial \Omega_{0}$-component of $\mathbb{X}(\Gamma)$ has been split into traces on $\partial \Omega_{1}$ and $\partial \Omega_{2}$.

Further, for the sake of lucidity, we only consider uniform permeabilities $\mu_{0}=\mu_{1}=\mu_{2}=1$ (and will suppress them in the notations in the sequel). Then, thanks to (5.3) the single-trace formulation (5.1) for this special situation can be recast as

$$
\left(\frac{1}{2} \mathrm{Id}-\mathrm{C}_{\kappa_{0}}^{0}\right)\left(\begin{array}{l}
-\mathbf{U}_{1} \\
-\mathbf{U}_{2}
\end{array}\right)=\left(\begin{array}{l}
-\gamma^{1}\left(u_{\mathrm{inc}}\right) \\
-\gamma^{2}\left(u_{\mathrm{inc}}\right)
\end{array}\right), \quad\left(\frac{1}{2} \mathrm{Id}-\mathrm{C}_{\kappa_{1}}^{1}\right) \mathbf{U}_{1}=0, \quad\left(\frac{1}{2} \mathrm{Id}-\mathrm{C}_{\kappa_{2}}^{2}\right) \mathbf{U}_{2}=0 .
$$

The splitting of $\mathbb{H}\left(\partial \Omega_{0}\right)$ induces a splitting of the first boundary integral equation in (5.4):

$$
\left(\frac{1}{2} \operatorname{Id}-\mathrm{C}_{\kappa_{0}}^{0}\right)\left(\begin{array}{c}
\mathbf{U}_{1} \\
\mathbf{U}_{2}
\end{array}\right)=\left(\begin{array}{cc}
\frac{1}{2} \mathrm{Id}+\mathrm{C}_{\kappa_{0}}^{1} & \mathrm{R}_{\kappa_{0}}^{1,2} \\
\mathrm{R}_{\kappa_{0}}^{2,1} & \frac{1}{2} \mathrm{Id}+\mathrm{C}_{\kappa_{0}}^{2}
\end{array}\right)\left(\begin{array}{c}
\mathbf{U}_{1} \\
\mathbf{U}_{2}
\end{array}\right)=\left(\begin{array}{c}
\gamma^{1}\left(u_{\text {inc }}\right) \\
\gamma^{2}\left(u_{\text {inc }}\right)
\end{array}\right),
$$

where the operators

$$
\mathrm{R}_{\kappa_{0}}^{2,1}:=\gamma^{2} \mathbf{G}_{\kappa_{0}}^{1}: \mathbb{H}\left(\partial \Omega_{1}\right) \rightarrow \mathbb{H}\left(\partial \Omega_{2}\right) \quad \text { and } \quad \mathrm{R}_{\kappa_{0}}^{1,2}:=\gamma^{1} \mathbf{G}_{\kappa_{0}}^{2}: \mathbb{H}\left(\partial \Omega_{2}\right) \rightarrow \mathbb{H}\left(\partial \Omega_{1}\right)
$$



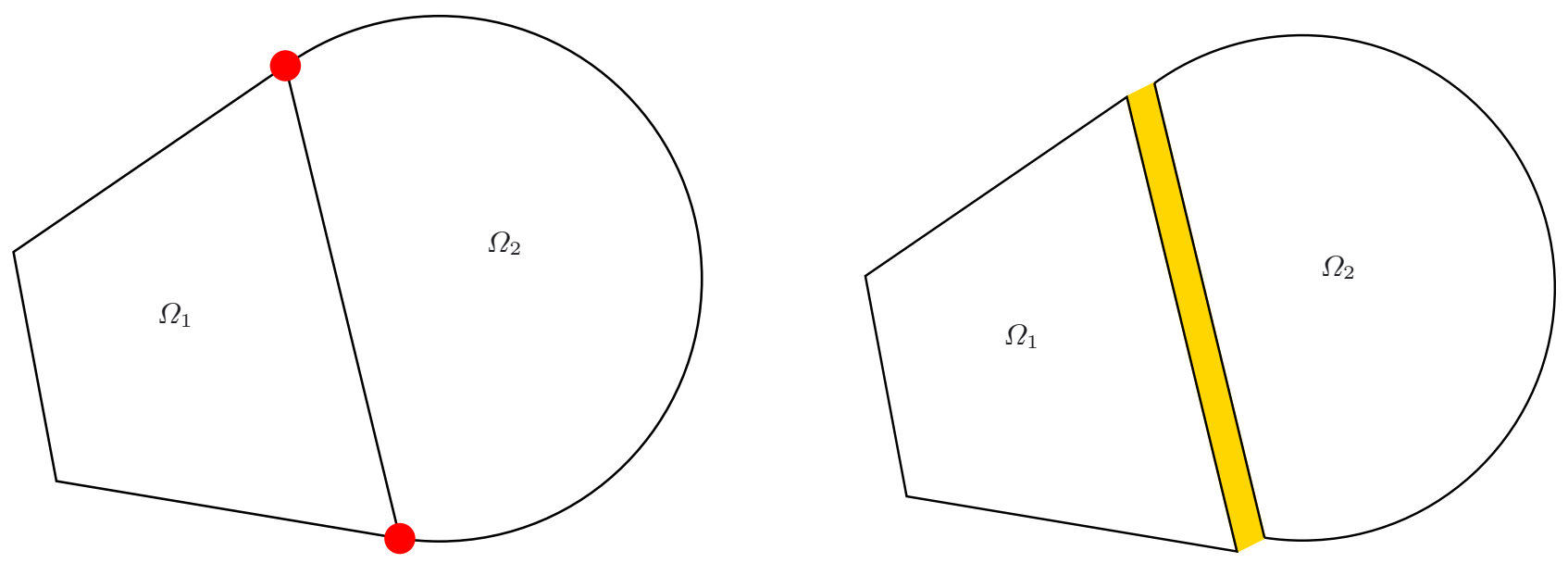

FIGURE 2. Introducing a virtual gap (colored in yellow) separating subdomains.

take into account the coupling between the different parts of $\partial \Omega_{0}$. Then we can merge the equations of (5.4) into one final single-trace formulation

$$
\left(\begin{array}{cc}
\mathrm{C}_{\kappa_{1}}^{1}+\mathrm{C}_{\kappa_{0}}^{1} & \mathrm{R}_{\kappa_{0}}^{1,2} \\
\mathrm{R}_{\kappa_{0}}^{2,1} & \mathrm{C}_{\kappa_{1}}^{2}+C_{\kappa_{0}}^{2}
\end{array}\right)\left(\begin{array}{c}
\mathbf{U}_{1} \\
\mathbf{U}_{2}
\end{array}\right)=\left(\begin{array}{c}
\gamma^{1}\left(u_{\mathrm{inc}}\right) \\
\gamma^{2}\left(u_{\mathrm{inc}}\right)
\end{array}\right) .
$$

Starting from (5.7) we motivate our new multi-trace formulation by means of a gap idea, cf. [19], Section 5. As above, for the sake of clarity, the case $n=2$ with adjacent $\Omega_{1}, \Omega_{2}$ will be considered in the remainder of this section. We imagine that we tear apart $\Omega_{1}$ and $\Omega_{2}$, thus opening up a narrow "virtual gap" into which $\Omega_{0}$ can intrude, see Figure 2 (right).

When (5.7) is applied in the situation with a gap of non-zero width, the integral operators comprising $\mathrm{R}_{\kappa_{0}}^{1,2}$ and $\mathrm{R}_{\kappa_{0}}^{2,1}$ feature analytic kernels. However, the crucial observation, readily seen from the definition (5.6) is that these integral operators remain meaningful even when the gap is shrunk to "width zero". Hence we can apply the single-trace formulation in the form (5.7) to the original situation of Figure 2, left. The unknown traces will be sought in $\mathbb{H}\left(\partial \Omega_{1}\right) \times \mathbb{H}\left(\Omega_{2}\right)$, which means that

- the unknowns on $\partial \Omega_{0}$ are a single pair of traces;

- on $\partial \Omega_{1} \cap \partial \Omega_{2}$ there are two pairs of unknown traces.

For this reason we have dubbed the new formulation, our new interpretation on (5.7) "multi-trace".

\section{Remarkable properties of the space of Cauchy data}

Parallel to [19], Section 6, in this section, we point out several properties of the Cauchy data spaces $\mathcal{C}_{\kappa_{j}, \mu_{j}}\left(\partial \Omega_{j}\right)$. The most important is probably that $\mathcal{C}_{\kappa, \mu}(\Gamma)$ provides a complement to $\mathbb{X}(\Gamma)$ in $\mathbb{H}(\Gamma)$ for any value of $\kappa, \mu \in(0,+\infty)$.

Proposition 6.1. Consider any $\mu_{j}, \kappa_{j} \in(0,+\infty), j=0 \ldots n$. Then we have the decomposition

$$
\mathbb{H}(\Gamma)=\mathbb{X}(\Gamma) \oplus \mathcal{C}_{\kappa, \mu}(\Gamma) .
$$

We do not provide the proof for this result because it is nearly the same as the proof to Proposition 6.1 in [19]. Next we state a characterization of Cauchy data along the lines of Proposition 3.1.

Lemma 6.2. For any $\mathbf{U} \in \mathbb{H}(\Gamma)$ we have: $\mathbf{U} \in \mathcal{C}_{\kappa, \mu}(\Gamma) \Longleftrightarrow \mathrm{B}(\mathbf{U}, \mathbf{V})=0, \forall \mathbf{V} \in \mathcal{C}_{\kappa, \mu}(\Gamma)$. 
Proof. To show this, we may proceed as in [19], Lemma 6.1. We propose here a different proof that is straightforward. Recall that Range $\left(\mathrm{Id} / 2+\mathrm{A}_{\kappa, \mu}\right)=\operatorname{Ker}\left(-\mathrm{Id} / 2+\mathrm{A}_{\kappa, \mu}\right)=\mathcal{C}_{\kappa, \mu}(\Gamma)$. Using Lemma 4.5, we see that for any $\mathbf{U} \in \mathbb{H}(\Gamma)$, we have $\mathbf{U} \in \mathfrak{C}_{\kappa, \mu}(\Gamma)$ if and only if

$$
\begin{aligned}
\mathrm{B}\left(\left(-\mathrm{Id} / 2+\mathrm{A}_{\kappa, \mu}\right) \mathbf{U}, \mathbf{V}\right)=0 & \forall \mathbf{V} \in \mathbb{H}(\Gamma) \\
& \Longleftrightarrow \mathrm{B}\left(\mathbf{U},\left(\mathrm{Id} / 2+\mathrm{A}_{\kappa, \mu}\right) \mathbf{V}\right)=0 \quad \forall \mathbf{V} \in \mathbb{H}(\Gamma) \\
& \Longleftrightarrow \mathrm{B}(\mathbf{U}, \mathbf{V})=0 \quad \forall \mathbf{V} \in \mathcal{C}_{\kappa, \mu}(\Gamma) .
\end{aligned}
$$

An immediate consequence of the previous lemma is that $\mathbf{U}_{j} \in \mathcal{C}_{\kappa_{j}, \mu_{j}}\left(\partial \Omega_{j}\right)$ if and only if $\mathrm{B}_{j}\left(\mathbf{U}_{j}, \mathbf{V}_{j}\right)=0, \forall \mathbf{V}_{j} \in$ $\mathcal{C}_{\kappa_{j}, \mu_{j}}\left(\partial \Omega_{j}\right)$. It is straightforward to adapt the proof of Lemma 6.2 so as to prove the following result.

Lemma 6.3. Let $j=0 \ldots n$ and take any $\kappa_{j}, \mu_{j} \in(0,+\infty)$. For any $\mathbf{U}_{j} \in \mathbb{H}\left(\partial \Omega_{j}\right)$, we have $\mathbf{U}_{j} \in$ Range $\left(-\mathrm{Id} / 2+\mathrm{A}_{\kappa_{j}, \mu_{j}}\right)$ if and only if $\mathrm{B}_{j}\left(\mathbf{U}_{j}, \mathbf{V}_{j}\right)=0 \forall \mathbf{V}_{j} \in \operatorname{Range}\left(-\mathrm{Id} / 2+\mathrm{A}_{\kappa_{j}, \mu_{j}}\right)$.

\section{NeW FunCtional SETting}

Following the same approach as in [19], Section 7, we introduce a new functional setting adapted to traces of functions restricted to $\mathbb{R}^{3} \backslash \bar{\Omega}_{0}$. We set

$$
\begin{aligned}
& \widehat{\mathbb{H}}(\Gamma)=\Pi_{j=1}^{n} \mathbb{H}\left(\partial \Omega_{j}\right) \\
& \widehat{\mathcal{C}}_{0}(\Gamma)=\Pi_{j=1}^{n} \mathcal{C}_{\kappa_{0}, \mu_{0}}\left(\partial \Omega_{j}\right) \\
& \widehat{\mathbb{X}}(\Gamma)=\left\{\left(\gamma^{j}(\mathbf{U})\right)_{j=1, \ldots n} \in \widehat{\mathbb{H}}(\Gamma) \mid \mathbf{U} \in \boldsymbol{H}_{\mathrm{loc}}\left(\operatorname{curl}^{2}, \mathbb{R}^{3}\right)\right\} .
\end{aligned}
$$

Note that the space $\widehat{\mathbb{H}}(\Gamma)$ differs from $\mathbb{H}(\Gamma)$ as the index $j$ in its definition ranges from 1 to $n$ (not from 0 to $n$ ). Moreover, notice that in the definition of $\widehat{\complement}_{0}(\Gamma)$, all wave numbers are equal to $\kappa_{0}$, and that only $\mu_{0}$ is involved (and not $\mu_{j}$ for $j \neq 0$ ). It is clear from (6.1) and (7.1) considered in the case $\kappa_{j}=\kappa_{0}, \forall j$ that

$$
\widehat{\mathbb{X}}(\Gamma)+\widehat{\mathfrak{C}}_{0}(\Gamma)=\widehat{\mathbb{H}}(\Gamma) .
$$

The sum above is not a direct sum as $\widehat{\mathbb{X}}(\Gamma) \cap \widehat{\complement}_{0}(\Gamma) \neq\{0\}$. We equip the space $\widehat{\mathbb{H}}(\Gamma)$ with a norm denoted \|\| , and a duality pairing analogous to the one considered for $\mathbb{H}(\Gamma)$, setting

$$
\begin{aligned}
\|\widehat{\mathbf{U}}\| & =\left(\sum_{j=1}^{n}\left\|\mathbf{u}_{j}\right\|_{\boldsymbol{H}^{-1 / 2}(\operatorname{div}, \partial \Omega)}^{2}+\left\|\mathbf{p}_{j}\right\|_{\boldsymbol{H}^{-1 / 2}(\operatorname{div}, \partial \Omega)}^{2}\right)^{\frac{1}{2}} \quad \text { for } \widehat{\mathbf{U}}=\left(\begin{array}{c}
\mathbf{u}_{j} \\
\mathbf{p}_{j}
\end{array}\right)_{j=1 \ldots n} \in \widehat{\mathbb{H}}(\Gamma) . \\
\widehat{\mathrm{B}}(\mathbf{U}, \mathbf{V}) & =\sum_{j=1}^{n} \mathrm{~B}_{j}\left(\mathbf{U}_{j}, \mathbf{V}_{j}\right) \quad \text { for } \mathbf{U}, \mathbf{V} \in \widehat{\mathbb{H}}(\Gamma) .
\end{aligned}
$$

Although $\widehat{\mathbb{X}}(\Gamma)$ may seem "smaller" than $\mathbb{X}(\Gamma)$ at first glance, both spaces are actually isomorphic, as pointed out by the following lemma.

Lemma 7.1. For any $\widehat{\mathbf{U}} \in \widehat{\mathbb{X}}(\Gamma)$, there exists a unique $\mathbf{U}_{0} \in \mathbb{H}\left(\partial \Omega_{0}\right)$ such that $\left(\mathbf{U}_{0}, \widehat{\mathbf{U}}\right) \in \mathbb{X}(\Gamma)$.

Proof. The existence is clear, what has to be proved here is the uniqueness of $\mathbf{U}_{0}$. Assume that for $\mathbf{U}_{0}, \mathbf{V}_{0} \in \mathbb{H}\left(\partial \Omega_{0}\right)$ we have $\left(\mathbf{U}_{0}, \widehat{\mathbf{U}}\right) \in \mathbb{X}(\Gamma)$ and $\left(\mathbf{V}_{0}, \widehat{\mathbf{U}}\right) \in \mathbb{X}(\Gamma)$ so that $\mathbf{U}:=\left(\mathbf{U}_{0}-\mathbf{V}_{0}, 0, \ldots, 0\right) \in \mathbb{X}(\Gamma)$. Consider $\mathbf{u}_{0}, \mathbf{p}_{0} \in \boldsymbol{H}^{-\frac{1}{2}}\left(\operatorname{div}, \partial \Omega_{0}\right)$ such that $\mathbf{U}_{0}-\mathbf{V}_{0}=\left(\mathbf{u}_{0}, \mathbf{p}_{0}\right)^{\top}$. Let us show that $\mathbf{u}_{0}=0$. Take any 
$\mathbf{w}_{0} \in \boldsymbol{H}^{-\frac{1}{2}}\left(\operatorname{div}, \partial \Omega_{0}\right)$, and choose $\mathbf{w}_{j} \in \boldsymbol{H}^{-\frac{1}{2}}\left(\operatorname{div}, \partial \Omega_{j}\right), j=0 \ldots n$ such that $\left(\mathbf{w}_{j}\right)_{j=0 \ldots n} \in \boldsymbol{X}(\Gamma)$ so that $\mathbf{W}=\left(0, \mathbf{w}_{j}\right)_{j=0 \ldots n}^{\top}$ belongs to $\mathbb{X}(\Gamma)$. We have

$$
\left\langle\mathbf{u}_{0}, \mathbf{w}_{0}\right\rangle_{\times, \partial \Omega_{0}}=\mathrm{B}(\mathbf{U}, \mathbf{W})=0
$$

since both $\mathbf{U}$ and $\mathbf{W}$ belong to $\mathbb{X}(\Gamma)$. Since $\mathbf{w}_{0}$ was chosen arbitrarily, we obtain that $\mathbf{u}_{0}=0$. We prove in the same manner that $\mathbf{p}_{0}=0$. This finally shows that $\mathbf{U}_{0}=\mathbf{V}_{0}$.

We will also need a weak characterization of the space $\widehat{\mathbb{X}}(\Gamma)$. Although $\mathbb{X}(\Gamma)$ is its own polar set according to Proposition 3.1, such is not the case for $\widehat{\mathbb{X}}(\Gamma)$.

Proposition 7.2. Let $\widehat{\mathbb{X}}_{0}(\Gamma)=\{\widehat{\mathbf{V}} \in \widehat{\mathbb{X}}(\Gamma) \mid(0, \widehat{\mathbf{V}}) \in \mathbb{X}(\Gamma)\}$. For any $\widehat{\mathbf{U}} \in \widehat{\mathbb{H}}(\Gamma)$ we have

$$
\widehat{\mathbf{U}} \in \widehat{\mathbb{X}}(\Gamma) \quad \Longleftrightarrow \quad \widehat{\mathrm{B}}(\widehat{\mathbf{U}}, \widehat{\mathbf{V}})=0 \quad \forall \widehat{\mathbf{V}} \in \widehat{\mathbb{X}}_{0}(\Gamma) .
$$

Proof. Assume first that $\widehat{\mathbf{U}} \in \widehat{\mathbb{X}}(\Gamma)$ and consider any $\widehat{\mathbf{V}} \in \widehat{\mathbb{X}}_{0}(\Gamma)$. Take $\mathbf{U}_{0} \in \mathbb{H}\left(\partial \Omega_{0}\right)$ such that $\left(\mathbf{U}_{0}, \widehat{\mathbf{U}}\right) \in \mathbb{X}(\Gamma)$ and set $\mathbf{V}=(\mathbf{0}, \widehat{\mathbf{V}}) \in \mathbb{X}(\Gamma)$. Applying Proposition 3.1, we have $\widehat{\mathrm{B}}(\widehat{\mathbf{U}}, \widehat{\mathbf{V}})=\mathrm{B}(\mathbf{U}, \mathbf{V})=0$.

Now assume that $\widehat{\mathbf{U}}=\left(\mathbf{u}_{j}, \mathbf{p}_{j}\right)_{j=1 \ldots n}^{\top} \in \widehat{\mathbb{H}}(\Gamma)$ satisfies the condition in the right hand side of (7.2). Let us show that $\left(\mathbf{u}_{0}, \mathbf{u}_{1}, \ldots, \mathbf{u}_{n}\right) \in \boldsymbol{X}(\Gamma)$ for some $\mathbf{u}_{0} \in \boldsymbol{H}^{-1 / 2}\left(\operatorname{div}, \partial \Omega_{0}\right)$. Take $\mathbf{v} \in \boldsymbol{L}_{\text {loc }}^{2}\left(\mathbb{R}^{3} \backslash \bar{\Omega}_{0}\right)$ such that $\left.\mathbf{v}\right|_{\Omega_{j}} \in \boldsymbol{H}_{\text {loc }}\left(\operatorname{curl}^{2}, \bar{\Omega}_{j}\right)$ and $\boldsymbol{\gamma}_{\mathrm{D}}^{j}(\mathbf{v})=\mathbf{u}_{j}, j=1 \ldots n$. Take any $\mathbf{w} \in \boldsymbol{H}_{\text {loc }}\left(\mathbf{c u r l}, \mathbb{R}^{3}\right)$ such that $\boldsymbol{\gamma}_{\mathrm{D}}^{0}(\mathbf{w})=0$. Set $\widehat{\mathbf{W}}=\left(\mathbf{0}, \boldsymbol{\gamma}_{\mathrm{D}}^{j}(\mathbf{w})\right)_{j=1 \ldots n}^{\top}$ and observe that $\widehat{\mathbf{W}} \in \widehat{\mathbb{X}}_{0}(\Gamma)$. Then we have

$$
\int_{\mathbb{R}^{3} \backslash \bar{\Omega}_{0}} \mathbf{v} \cdot \operatorname{curl}(\mathbf{w}) \mathrm{d} \mathbf{x}-\sum_{j=1}^{n} \int_{\Omega_{j}} \mathbf{w} \cdot \operatorname{curl}(\mathbf{v}) \mathrm{d} \mathbf{x}=\widehat{\mathrm{B}}(\widehat{\mathbf{U}}, \widehat{\mathbf{W}})=0
$$

Since this holds for any $\mathbf{w} \in \boldsymbol{H}_{\text {loc }}\left(\mathbf{c u r l}, \mathbb{R}^{3}\right)$ such that $\boldsymbol{\gamma}_{\mathrm{D}}^{0}(\mathbf{w})=0$, we conclude that $\mathbf{v} \in \boldsymbol{H}_{\text {loc }}\left(\operatorname{curl}, \mathbb{R}^{3} \backslash \bar{\Omega}_{0}\right)$. Extending $\mathbf{v}$ properly, using a continuous right inverse of $\boldsymbol{\gamma}_{\mathrm{D}}^{0}$, we can consider that $\mathbf{v} \in \boldsymbol{H}_{\text {loc }}\left(\mathbf{c u r l}, \mathbb{R}^{3}\right)$. Setting $\mathbf{u}_{0}=\gamma_{\mathrm{D}}^{0}(\mathbf{v})$ we obtain $\left(\mathbf{u}_{j}\right)_{j=0 \ldots n} \in \boldsymbol{X}(\Gamma)$. We prove in the same manner that there exists $\mathbf{p}_{0}$ such that $\left(\mathbf{p}_{0}, \mathbf{p}_{1} \ldots, \mathbf{p}_{n}\right) \in \boldsymbol{X}(\Gamma)$. Setting $\mathbf{U}_{0}=\left(\mathbf{u}_{0}, \mathbf{p}_{0}\right)^{\top} \in \mathbb{H}\left(\partial \Omega_{0}\right)$, we finally have $\left(\mathbf{U}_{0}, \widehat{\mathbf{U}}\right) \in \mathbb{X}(\Gamma)$.

\section{NEW FORMULATION OF THE FIRST KIND}

In the present section, we state a reformulation of problem (5.2). To justify such a new formulation, the proof is nearly exactly the same as in Sections 7-9 of [19] (even notations coincide), so we do not give a lot of details. The only specific point, in the case of Maxwell's equations compared to the acoustic scattering problem, is the proof of Lemma 4.2 of the present article, that is different from the proof of Lemma 8.1 in [19].

To state this new formulation, we have to introduce a "transformed right hand side" and a "transformed operator". Following [19], we set

$$
\begin{aligned}
\widehat{\mathbf{F}}^{\text {inc }}:=-\left(\mathbf{F}_{j}^{\mathrm{inc}}+\mathcal{T}_{\mu_{0}} \cdot \gamma^{j} \cdot \mathbf{G}_{\kappa_{0}}^{0}\left(\mathcal{T}_{\mu_{0}}^{-1}\left(\mathbf{F}_{0}^{\mathrm{inc}}\right)\right)\right)_{j=1 \ldots n} \\
\text { with } \quad \mathbf{F}^{\text {inc }}=\left(\mathbf{F}_{j}^{\text {inc }}\right)_{j=0 \ldots n} \in \operatorname{Range}\left(-\mathrm{Id} / 2+\mathrm{A}_{\kappa, \mu}\right) .
\end{aligned}
$$


As in [19], we emphasize that the analysis underlying the formulation that we introduce do not depend on the specific form of $\widehat{\mathbf{F}}^{\text {inc }}$. For any $\mathbf{U}=\left(\mathbf{U}_{1}, \ldots, \mathbf{U}_{n}\right) \in \widehat{\mathbb{H}}(\Gamma)$, we define

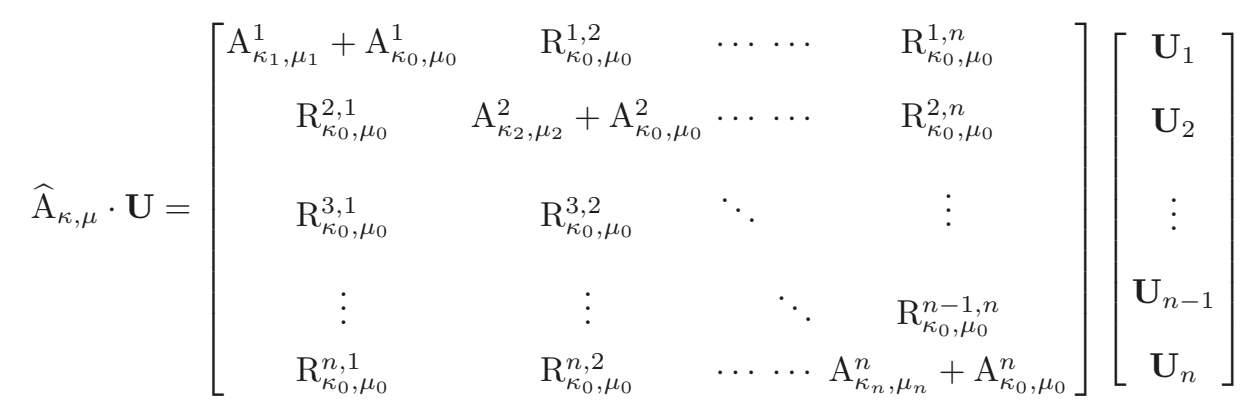

where we have set

$$
\mathrm{R}_{\kappa, \mu}^{q, j} \stackrel{\text { def }}{=} \mathcal{T}_{\mu} \cdot \gamma^{q} \cdot \mathbf{G}_{\kappa}^{j} \cdot \mathcal{T}_{\mu}^{-1} .
$$

Clearly, the operators $\mathrm{R}_{\kappa_{0}, \mu_{0}}^{q, j}$ are continuous maps from $\mathbb{H}\left(\partial \Omega_{j}\right)$ into $\mathbb{H}\left(\partial \Omega_{q}\right)$. Note that, as a consequence of Theorem 4.1, we have $\mathrm{R}_{\kappa_{0}, \mu_{0}}^{q, j} \cdot\left(\operatorname{Id} / 2+\mathrm{A}_{\kappa_{0}, \mu_{0}}^{j}\right)=0$ and $\left(\mathrm{Id} / 2-\mathrm{A}_{\kappa_{0}, \mu_{0}}^{q}\right) \cdot \mathrm{R}_{\kappa_{0}, \mu_{0}}^{q, j}=0$ as well as $\mathrm{R}_{\kappa_{0}, \mu_{0}}^{q, j} \cdot \mathrm{R}_{\kappa_{0}, \mu_{0}}^{j, p}=0$ whenever $q \neq j$. With the previous notations, a derivation identical to Sections 7-9 of [19] leads to the following theorem.

Theorem 8.1. Assume that $\mathbf{F}^{\text {inc }}$ and $\widehat{\mathbf{F}}^{\text {inc }}$ satisfy equation (8.1). In this case $\mathbf{U}=\left(\mathbf{U}_{0}, \widehat{\mathbf{U}}\right) \in \mathbb{H}(\Gamma)$ is solution to (5.2) if and only if $\widehat{\mathbf{U}}$ is solution to the problem

$$
\text { Find } \widehat{\mathbf{U}} \in \widehat{\mathbb{H}}(\Gamma) \text { such that } \widehat{\mathrm{B}}\left(\widehat{\mathrm{A}}_{\kappa, \mu}(\widehat{\mathbf{U}}), \widehat{\mathbf{V}}\right)=\widehat{\mathrm{B}}\left(\widehat{\mathbf{F}}^{\mathrm{inc}}, \widehat{\mathbf{V}}\right) \quad \forall \widehat{\mathbf{V}} \in \widehat{\mathbb{H}}(\Gamma) \text {. }
$$

The proof of this theorem is identical to the proof of Theorems 8.1 and 8.2 in [19]. A remarkable feature of formulation (8.4) is that it is posed in $\widehat{\mathbb{H}}(\Gamma)$ and not in $\widehat{\mathbb{X}}(\Gamma)$. In other words, this new variational setting does not contain any constraint.

We end this section by proving a symmetry property satisfied by the operator $\widehat{\mathrm{A}}_{\kappa, \mu}$, that is, the multisubdomain counterpart of Theorem 9 in [13]. This property will be useful in the next section.

Proposition 8.2 (symmetry of multi-trace operator). For any $\kappa_{0}, \kappa_{1}, \ldots, \kappa_{n} \in(0,+\infty)$ and any $\mu_{0}, \mu_{1}, \ldots, \mu_{n} \in(0,+\infty)$ we have the following symmetry property

$$
\widehat{\mathrm{B}}\left(\widehat{\mathrm{A}}_{\kappa, \mu}(\mathbf{U}), \mathbf{V}\right)=\widehat{\mathrm{B}}\left(\widehat{\mathrm{A}}_{\kappa, \mu}(\mathbf{V}), \mathbf{U}\right) \quad \forall \mathbf{U}, \mathbf{V} \in \widehat{\mathbb{H}}(\Gamma) .
$$

Proof. We know that $\mathrm{B}_{j}\left(\mathrm{~A}_{\kappa_{j}, \mu_{j}}\left(\mathbf{U}_{j}\right), \mathbf{V}_{j}\right)=\mathrm{B}_{j}\left(\mathrm{~A}_{\kappa_{j}, \mu_{j}}\left(\mathbf{V}_{j}\right), \mathbf{U}_{j}\right)$ for any $\mathbf{U}_{j}, \mathbf{V}_{j} \in \mathbb{H}\left(\partial \Omega_{j}\right)$ and any value of $\kappa_{j}, \mu_{j} \in(0,+\infty)$, see the proof of Lemma 4.5. As a consequence, the result will be proved if we can show that

$$
\mathrm{B}_{j}\left(\mathrm{R}_{\kappa_{0}, \mu_{0}}^{j, q}\left(\mathbf{U}_{q}\right), \mathbf{V}_{j}\right)=\mathrm{B}_{q}\left(\mathrm{R}_{\kappa_{0}, \mu_{0}}^{q, j}\left(\mathbf{V}_{j}\right), \mathbf{U}_{q}\right) \quad \text { for } j \neq q .
$$

Since $\mathrm{B}_{j}\left(\mathrm{R}_{\kappa_{0}, \mu_{0}}^{j, q}\left(\mathbf{U}_{q}\right), \mathbf{V}_{j}\right)=\mu_{0}^{-1} \mathrm{~B}_{j}\left(\gamma^{j} \mathbf{G}_{\kappa_{0}}^{q}\left(\mathcal{T}_{\mu_{0}}^{-1} \mathbf{U}_{q}\right), \mathcal{T}_{\mu_{0}}^{-1} \mathbf{V}_{j}\right)$, it suffices to show the following identity

$$
\mathrm{B}_{j}\left(\boldsymbol{\gamma}^{j} \cdot \mathbf{G}_{\kappa_{0}}^{q}\left(\mathbf{U}_{q}\right), \mathbf{V}_{j}\right)=\mathrm{B}_{q}\left(\gamma^{q} \cdot \mathbf{G}_{\kappa_{0}}^{j}\left(\mathbf{V}_{j}\right), \mathbf{U}_{q}\right) \quad \forall \mathbf{U}_{q} \in \mathbb{H}\left(\partial \Omega_{q}\right), \forall \mathbf{V}_{j} \in \mathbb{H}\left(\partial \Omega_{j}\right)
$$

for any $j, q \in\{1, \ldots n\}$ with $j \neq q$. Hence pick arbitrary $j, q \in\{1, \ldots n\}$ with $j \neq q$ and $\mathbf{U}_{q} \in \mathbb{H}\left(\partial \Omega_{q}\right)$, $\mathbf{V}_{j} \in \mathbb{H}\left(\partial \Omega_{j}\right)$. Consider $\mathbf{W}^{j}, \mathbf{W}^{q} \in \mathbb{X}(\Gamma)$ defined by $\mathbf{W}^{\alpha}=\left(\mathbf{W}_{j}^{\alpha}\right)_{j=0 \ldots n}$ for $\alpha=j, q$ with

$$
\begin{aligned}
& \mathbf{W}_{q}^{q}=\boldsymbol{\gamma}_{c}^{q} \cdot \mathbf{G}_{\kappa_{0}}^{q}\left(\mathbf{U}_{q}\right) \text { and } \quad \mathbf{W}_{k}^{q}=\boldsymbol{\gamma}^{k} \cdot \mathbf{G}_{\kappa_{0}}^{q}\left(\mathbf{U}_{q}\right) \text { for } k \neq q, \\
& \mathbf{W}_{j}^{j}=\gamma_{c}^{j} \cdot \mathbf{G}_{\kappa_{0}}^{j}\left(\mathbf{V}_{j}\right) \text { and } \mathbf{W}_{k}^{j}=\boldsymbol{\gamma}^{k} \cdot \mathbf{G}_{\kappa_{0}}^{j}\left(\mathbf{V}_{j}\right) \text { for } k \neq j .
\end{aligned}
$$


We have $\mathbf{V}_{j}=\left[\boldsymbol{\gamma}^{j}\right] \cdot \mathbf{G}_{\kappa_{0}}^{j}\left(\mathbf{V}_{j}\right)$ according to the jump formula (4.2). Using this identity, as well as Lemma 6.2, we obtain

$$
\begin{aligned}
\mathrm{B}_{j}\left(\boldsymbol{\gamma}^{j} \cdot \mathbf{G}_{\kappa_{0}}^{q}\left(\mathbf{U}_{q}\right), \mathbf{V}_{j}\right) & =\mathrm{B}_{j}\left(\boldsymbol{\gamma}^{j} \cdot \mathbf{G}_{\kappa_{0}}^{q}\left(\mathbf{U}_{q}\right),\left[\boldsymbol{\gamma}^{j}\right] \cdot \mathbf{G}_{\kappa_{0}}^{j}\left(\mathbf{V}_{j}\right)\right) \\
& =-\mathrm{B}_{j}\left(\boldsymbol{\gamma}^{j} \cdot \mathbf{G}_{\kappa_{0}}^{q}\left(\mathbf{U}_{q}\right), \boldsymbol{\gamma}_{c}^{j} \cdot \mathbf{G}_{\kappa_{0}}^{j}\left(\mathbf{V}_{j}\right)\right) \stackrel{(8.7)}{=}-\mathrm{B}_{j}\left(\mathbf{W}_{j}^{q}, \mathbf{W}_{j}^{j}\right)
\end{aligned}
$$

As $\mathbf{W}^{j}, \mathbf{W}^{q} \in \mathbb{X}(\Gamma)$, we can apply Proposition 3.1, which yields

$$
-\mathrm{B}_{j}\left(\mathbf{W}_{j}^{q}, \mathbf{W}_{j}^{j}\right)=\mathrm{B}_{q}\left(\mathbf{W}_{q}^{q}, \mathbf{W}_{q}^{j}\right)+\sum_{\substack{k=0 \ldots n \\ k \neq j, q}} \mathrm{~B}_{k}\left(\mathbf{W}_{k}^{q}, \mathbf{W}_{k}^{j}\right) .
$$

We have $\mathbf{W}_{k}^{j}, \mathbf{W}_{k}^{q} \in \mathcal{C}_{\kappa_{0}}\left(\partial \Omega_{k}\right)$ for $k \in\{0 \ldots n\} \backslash\{j, q\}$, so that $\mathrm{B}_{k}\left(\mathbf{W}_{k}^{q}, \mathbf{W}_{k}^{j}\right)=0$ for $k \neq j, q$. As a consequence, we can apply Lemma 6.2 , and since $\mathbf{U}_{q}=\left[\boldsymbol{\gamma}^{q}\right] \cdot \mathbf{G}_{\kappa_{0}}^{q}\left(\mathbf{U}_{q}\right)$, we obtain

$$
\begin{aligned}
\mathrm{B}_{j}\left(\boldsymbol{\gamma}^{j} \cdot \mathbf{G}_{\kappa_{0}}^{q}\left(\mathbf{U}_{q}\right), \mathbf{V}_{j}\right) & =\mathrm{B}_{q}\left(\gamma_{c}^{q} \cdot \mathbf{G}_{\kappa_{0}}^{q}\left(\mathbf{U}_{q}\right), \gamma^{q} \cdot \mathbf{G}_{\kappa_{0}}^{j}\left(\mathbf{V}_{j}\right)\right) \\
& =-\mathrm{B}_{q}\left(\left[\gamma^{q}\right] \cdot \mathbf{G}_{\kappa_{0}}^{q}\left(\mathbf{U}_{q}\right), \gamma^{q} \cdot \mathbf{G}_{\kappa_{0}}^{j}\left(\mathbf{V}_{j}\right)\right)=\mathrm{B}_{q}\left(\gamma^{q} \cdot \mathbf{G}_{\kappa_{0}}^{j}\left(\mathbf{V}_{j}\right), \mathbf{U}_{q}\right),
\end{aligned}
$$

which concludes the proof.

\section{Coercivity}

The coercivity property for problem (8.4), which we tackle now, is more involved in the case of Maxwell's equations than in the case of acoustic scattering. As a consequence, we shall give much more details here.

\subsection{Splitting of the variational space}

The proof of coercivity for first kind integral formulations in the case of Maxwell's equations developed in [13] relies on a key splitting idea, which was pioneered in $[15,24,27]$. We will adopt a similar approach in the present case. First, let us recall the following result that was established in [10], Lemma 2.

Lemma 9.1 (regular decomposition of trace space). For any Lipschitz open set $\Omega \subset \mathbb{R}^{3}$, there exists a subspace $z(\partial \Omega) \subset \boldsymbol{H}_{\times}^{1 / 2}(\partial \Omega)$ that is closed in $\boldsymbol{H}^{-1 / 2}(\operatorname{div}, \partial \Omega)$ and gives a direct sum decomposition

$$
z(\partial \Omega) \oplus \mathcal{N}(\partial \Omega)=\boldsymbol{H}^{-\frac{1}{2}}(\operatorname{div}, \partial \Omega), \quad \text { where } \mathcal{N}(\partial \Omega):=\left\{\mathbf{q} \in \boldsymbol{H}^{-\frac{1}{2}}(\operatorname{div}, \partial \Omega) \mid \operatorname{div} \partial \Omega(\mathbf{q})=0\right\} .
$$

Note that, since the injection $\boldsymbol{H}_{\times}^{1 / 2}(\partial \Omega) \hookrightarrow \boldsymbol{H}_{\times}^{-1 / 2}(\partial \Omega)$ is compact, the bilinear form $(\mathbf{u}, \mathbf{v}) \mapsto\langle\mathbf{u}, \mathbf{v}\rangle_{\times, \partial \Omega}$ is compact when restricted to $z(\partial \Omega) \times z(\partial \Omega)$. Besides, since $z\left(\partial \Omega_{j}\right)$ is closed in $\boldsymbol{H}^{-1 / 2}\left(\operatorname{div}, \partial \Omega_{j}\right)$, for any $j=1 \ldots n$ there exists a continuous extension operator $\boldsymbol{\Phi}^{j}: \mathcal{Z}\left(\partial \Omega_{j}\right) \rightarrow \boldsymbol{H}_{\mathrm{loc}}^{1}\left(\bar{\Omega}_{j}\right)$ such that

$$
\boldsymbol{\gamma}_{\mathrm{D}}^{j} \cdot \boldsymbol{\Phi}^{j}(\mathbf{v})=\mathbf{v} \quad \forall \mathbf{v} \in Z\left(\partial \Omega_{j}\right) \quad \forall j=1 \ldots n .
$$

Such a decomposition as (9.1) should be understood in the sense that any element of $\boldsymbol{H}^{-1 / 2}(\operatorname{div}, \partial \Omega)$ can be decomposed in a regular trace (which will bring some compactness property in the forthcoming analysis) and a trace with vanishing surface divergence. We shall consider the cartesian product of pairs of such decompositions for all subdomains, writing

$$
\widehat{\mathbb{H}}(\Gamma)=\boldsymbol{Z}(\Gamma) \oplus \mathcal{N}(\Gamma) \quad \text { where } \quad \boldsymbol{Z}(\Gamma)=\prod_{j=1}^{n}\left(\mathcal{Z}\left(\partial \Omega_{j}\right)\right)^{2} \quad \text { and } \quad \mathcal{N}(\Gamma)=\prod_{j=1}^{n}\left(\mathcal{N}\left(\partial \Omega_{j}\right)\right)^{2}
$$


Define the projectors $\mathrm{P}_{\boldsymbol{z}}: \widehat{\mathbb{H}}(\Gamma) \rightarrow \boldsymbol{z}(\Gamma)$ such that $\operatorname{Ker}\left(\mathrm{P}_{\boldsymbol{z}}\right)=\mathcal{N}(\Gamma)$. In the sequel, following the example of [13], Theorem 3.12, we shall also simply write $\mathbf{U}^{\mathcal{Z}}=\mathrm{P}_{\mathcal{Z}}(\mathbf{U})$ and $\mathbf{U}^{\mathcal{N}}=\mathbf{U}-\mathrm{P}_{\mathcal{Z}}(\mathbf{U})$. Finally we define the operator $\Xi: \widehat{\mathbb{H}}(\Gamma) \rightarrow \widehat{\mathbb{H}}(\Gamma)$ by

$$
\Xi(\mathbf{U})=\overline{\mathbf{U}-2 \mathrm{P}_{\mathbf{z}}(\mathbf{U})}=\overline{\mathbf{U}^{\mathcal{N}}-\mathbf{U}^{\mathcal{Z}}} \text { for } \mathbf{U}=\mathbf{U}^{\mathcal{N}}+\mathbf{U}^{\mathcal{Z}} .
$$

Observe that $\Xi: \widehat{\mathbb{H}}(\Gamma) \rightarrow \widehat{\mathbb{H}}(\Gamma)$ is clearly a continuous isomorphism since it is an involution i.e. $\Xi^{2}=\mathrm{Id}$.

\subsection{Outline of the proof of coercivity}

Before delving into the proof of coercivity, we list a few useful observations. First of all, notice that the elements located on the diagonal of $\widehat{\mathrm{A}}_{\kappa, \mu}$ are operators associated with formulations of the first kind for transmission problems in the case of isolated scatterers. For each of these operators, coercivity has already been established. This suggests that we decompose $\widehat{\mathrm{A}}_{\kappa, \mu}$ in the following manner,

$$
\widehat{\mathrm{A}}_{\kappa, \mu}=\mathrm{D}_{\kappa, \mu}+\mathrm{R}_{\kappa_{0}, \mu_{0}} \quad \text { with } \quad \mathrm{D}_{\kappa, \mu}:=\left[\begin{array}{cccc}
\mathrm{A}_{\kappa_{1}, \mu_{1}}^{1} & 0 & \cdots & 0 \\
0 & \ddots & & \vdots \\
\vdots & & \ddots & 0 \\
0 & \cdots & 0 & \mathrm{~A}_{\kappa_{n}, \mu_{n}}^{n}
\end{array}\right] .
$$

The operator $\mathrm{R}_{\kappa_{0}, \mu_{0}}$ only depends on $\kappa_{0}, \mu_{0}$ (and not on $\kappa_{1}, \mu_{1}, \ldots, \kappa_{n}, \mu_{n}$ ). We already know from [13], Theorem 3.12, or [10], Theorem 9 , that $\mathrm{D}_{\kappa, \mu}$ satisfies a generalized Gårding inequality.

Theorem 9.2. There exists a compact operator $\mathrm{K}_{1}: \widehat{\mathbb{H}}(\Gamma) \rightarrow \widehat{\mathbb{H}}(\Gamma)$ and a constant $C>0$ such that

$$
\Re e\left\{\widehat{\mathrm{B}}\left(\left(\mathrm{D}_{\kappa, \mu}+\mathrm{K}_{1}\right) \mathbf{U}, \Xi(\mathbf{U})\right)\right\} \geq C\|\mathbf{U}\|^{2} \quad \forall \mathbf{U} \in \widehat{\mathbb{H}}(\Gamma) .
$$

In order to prove a similar result for $\widehat{\mathrm{A}}_{\kappa, \mu}$, we are going to show that $\mathrm{R}_{\kappa, \mu}$ satisfies a positivity property up to some compact perturbation: we are going to prove that there exists a compact operator $\mathrm{K}_{2}: \widehat{\mathbb{H}}(\Gamma) \rightarrow \widehat{\mathbb{H}}(\Gamma)$ such that

$$
\Re e\left\{\widehat{\mathrm{B}}\left(\left(\mathrm{R}_{\kappa_{0}, \mu_{0}}+\mathrm{K}_{2}\right) \mathbf{U}, \Xi(\mathbf{U})\right)\right\} \geq 0 \quad \forall \mathbf{U} \in \widehat{\mathbb{H}}(\Gamma) .
$$

Slightly abusing notations, denote $\mathrm{T}_{0}(\mathbf{U}):=\left(\mathcal{T}_{\mu_{0}}\left(\mathbf{U}_{1}\right), \ldots, \mathcal{T}_{\mu_{0}}\left(\mathbf{U}_{n}\right)\right)$ for any $\widehat{\mathbf{U}}=\left(\mathbf{U}_{1}, \ldots, \mathbf{U}_{n}\right) \in \widehat{\mathbb{H}}(\Gamma)$. Consider the operator $\mathrm{R}_{\kappa_{0}}$ defined in the same manner as $\mathrm{R}_{\kappa_{0}, \mu_{0}}$ but with $\mu_{0}=1$. Then we have $\mathrm{R}_{\kappa_{0}, \mu_{0}}=$ $\mathrm{T}_{0} \cdot \mathrm{R}_{\kappa_{0}} \cdot \mathrm{T}_{0}^{-1}$. Since $\mathrm{T}_{0} \cdot \Xi=\Xi \cdot \mathrm{T}_{0}$, we have

$$
\widehat{\mathrm{B}}\left(\mathrm{R}_{\kappa_{0}, \mu_{0}}(\mathbf{U}), \Xi(\mathbf{U})\right)=\mu_{0}^{-1} \cdot \widehat{\mathrm{B}}\left(\mathrm{R}_{\kappa_{0}}\left(\mathrm{~T}_{0}^{-1} \mathbf{U}\right), \Xi\left(\mathrm{T}_{0}^{-1} \mathbf{U}\right)\right) \quad \forall \mathbf{U} \in \widehat{\mathbb{H}}(\Gamma) .
$$

As a consequence, Estimate (9.2) holds if and only if there exists some compact operator $\mathrm{K}_{3}: \widehat{\mathbb{H}}(\Gamma) \rightarrow \widehat{\mathbb{H}}(\Gamma)$ such that

$$
\Re e\left\{\widehat{\mathrm{B}}\left(\left(\mathrm{R}_{\kappa_{0}}+\mathrm{K}_{3}\right) \mathbf{U}, \Xi(\mathbf{U})\right)\right\} \geq 0 \quad \forall \mathbf{U} \in \widehat{\mathbb{H}}(\Gamma) .
$$

\subsection{Perturbed potentials}

To further simplify the analysis, we need to introduce perturbed potentials by picking the imaginary "wave number" $\kappa=\boldsymbol{\imath}$ (imaginary unit). Note that $\mathscr{G}_{\boldsymbol{\imath}}(\mathbf{x})=\exp (-|\mathbf{x}|) /(4 \pi|\mathbf{x}|)$ is a positive real valued function 
exponentially decreasing for $|\mathbf{x}| \rightarrow \infty$. Consider the corresponding Newton potentials $\Psi_{\imath}$ and $\Psi_{\imath}$ defined by (4.1). Set

$$
\begin{aligned}
& \mathbf{S L}_{\boldsymbol{\imath}}^{j}(\mathbf{q})(\mathbf{x}):=\Psi_{\boldsymbol{\imath}}^{j}(\mathbf{q})(\mathbf{x})+\kappa_{0}^{-2} \nabla\left(\Psi_{\imath}^{j}\left(\operatorname{div}_{\partial \Omega_{j}}(\mathbf{q})\right)\right)(\mathbf{x}) \\
& \mathbf{D L}_{\boldsymbol{\imath}}^{j}(\mathbf{v})(\mathbf{x}):=\operatorname{curl}\left(\Psi_{\imath}^{j}(\mathbf{v})\right)(\mathbf{x}) \\
& \mathbf{G}_{\boldsymbol{\imath}}^{j}\left(\left[\begin{array}{l}
\mathbf{v} \\
\mathbf{q}
\end{array}\right]\right)(\mathbf{x})=\mathbf{D L}_{\imath}^{j}(\mathbf{v})(\mathbf{x})+\mathbf{S L}_{\imath}^{j}(\mathbf{q})(\mathbf{x}) \quad \forall \mathbf{v}, \mathbf{q} \in \boldsymbol{H}^{-\frac{1}{2}}\left(\operatorname{div}, \partial \Omega_{j}\right) .
\end{aligned}
$$

Observe that $\mathbf{S L}_{\boldsymbol{\imath}}^{j}(\mathbf{q})(\mathbf{x})$ still depends on $\kappa_{0}$. The only difference in the definition of $\mathbf{G}_{\boldsymbol{\imath}}^{j}$ compared to the definition of $\mathbf{G}_{\kappa_{0}}^{j}$ is that $\Psi_{\kappa_{0}}^{j}$ and $\Psi_{\kappa_{0}}^{j}$ have been replaced by $\Psi_{\boldsymbol{i}}^{j}, \boldsymbol{\Psi}_{\boldsymbol{\imath}}^{j}$. Then we consider the operator

$$
\mathrm{R}_{\imath} \cdot \mathbf{U}=\left[\begin{array}{cccc}
\left\{\gamma^{1}\right\} \cdot \mathbf{G}_{\imath}^{1} & \gamma^{1} \cdot \mathbf{G}_{\imath}^{2} & \cdots & \gamma^{1} \cdot \mathbf{G}_{\imath}^{n} \\
\gamma^{2} \cdot \mathbf{G}_{\imath}^{1} & \left\{\gamma^{2}\right\} \cdot \mathbf{G}_{\imath}^{2} \cdots & \gamma^{2} \cdot \mathbf{G}_{\imath}^{n} \\
\vdots & \vdots & \ddots & \vdots \\
\gamma^{n} \cdot \mathbf{G}_{\imath}^{1} & \gamma^{n} \cdot \mathbf{G}_{\imath}^{2} & \cdots & \left\{\gamma^{n}\right\} \cdot \mathbf{G}_{\boldsymbol{\imath}}^{n}
\end{array}\right]\left[\begin{array}{c}
\mathbf{U}_{1} \\
\mathbf{U}_{2} \\
\vdots \\
\mathbf{U}_{n}
\end{array}\right]
$$

Because of the regularity of $\mathscr{G}_{\kappa_{0}}(\mathbf{x})-\mathscr{G}_{\mathbf{\imath}}(\mathbf{x})$, we obtain that $\Psi_{\kappa_{0}}-\Psi_{\boldsymbol{\imath}}$ is an operator of order -4 that is continuous from $H^{1}\left(\mathcal{B}_{r}\right)^{\prime}$ to $H^{3}\left(\mathcal{B}_{r}\right)$ for any open ball $\mathcal{B}_{r}$ that contains $\cup_{j=1}^{n} \bar{\Omega}_{j}$, see [34], Remark 3.1.3. We deduce that $\mathrm{R}_{\kappa_{0}}-\mathrm{R}_{\imath}: \widehat{\mathbb{H}}(\Gamma) \rightarrow \widehat{\mathbb{H}}(\Gamma)$ is a compact operator. As a consequence, to show (9.3), it suffices to prove that there exists a compact operator $\mathrm{K}_{4}: \widehat{\mathbb{H}}(\Gamma) \rightarrow \widehat{\mathbb{H}}(\Gamma)$ such that

$$
\Re e\left\{\widehat{\mathrm{B}}\left(\left(\mathrm{R}_{\imath}+\mathrm{K}_{4}\right) \mathbf{U}, \Xi(\mathbf{U})\right)\right\} \geq 0 \quad \forall \mathbf{U} \in \widehat{\mathbb{H}}(\Gamma) .
$$

Let us take a closer look at the expression of $\widehat{\mathrm{B}}\left(\mathrm{R}_{\imath} \mathbf{U}, \Xi(\mathbf{U})\right)$, taking into account the definition of $\Xi$. We have

$$
\begin{aligned}
\Re e\left\{\widehat{\mathrm{B}}\left(\mathrm{R}_{\imath} \mathrm{U}, \Xi(\mathrm{U})\right)\right\} & =\Re e\left\{\widehat{\mathrm{B}}\left(\mathrm{R}_{\imath} \mathrm{U}^{\mathcal{N}}, \overline{\mathrm{U}^{\mathcal{N}}}\right)-\widehat{\mathrm{B}}\left(\mathrm{R}_{\imath} \mathrm{U}^{\mathcal{Z}}, \overline{\mathrm{U}^{\mathcal{Z}}}\right)\right\}+\Re e\left\{\widehat{\mathrm{B}}\left(\mathrm{R}_{\imath} \mathrm{U}^{\mathcal{Z}}, \overline{\mathrm{U}^{\mathcal{N}}}\right)-\widehat{\mathrm{B}}\left(\mathrm{R}_{\imath} \mathrm{U}^{\mathcal{N}}, \overline{\mathrm{U}^{\mathcal{Z}}}\right)\right\} \\
& =\Re e\left\{\widehat{\mathrm{B}}\left(\mathrm{R}_{\imath} \mathbf{U}^{\mathcal{N}}, \overline{\mathrm{U}^{\mathcal{N}}}\right)-\widehat{\mathrm{B}}\left(\mathrm{R}_{\imath} \mathrm{U}^{\mathcal{Z}}, \overline{\mathrm{U}^{\mathcal{Z}}}\right)\right\},
\end{aligned}
$$

because $\mathrm{R}_{\imath}$ commutes with complex conjugation (since the kernels of its integral operators are real) and enjoys symmetry with respect to the pairing $\widehat{\mathrm{B}}(\cdot, \cdot)$, which can be proved in exactly the same way as (8.5).

To deal with remaining terms, let us inspect in detail the expression of $\mathrm{B}\left(\mathrm{R}_{\imath} \mathbf{V}, \overline{\mathbf{V}}\right)$ for any $\mathbf{V} \in \widehat{\mathbb{H}}(\Gamma)$. If $\mathbf{V}=\left(\mathbf{v}_{j}, \mathbf{q}_{j}\right)_{j=1 \ldots n}^{\top}$ then we have

$$
\begin{aligned}
\mathrm{B}\left(\mathrm{R}_{\imath} \mathbf{V}, \overline{\mathbf{V}}\right)= & \sum_{i=1}^{n} \sum_{j=1}^{n}\left\langle\gamma_{\mathrm{D}}^{i} \cdot \mathbf{S L}_{\boldsymbol{\imath}}^{j}\left(\mathbf{q}_{j}\right), \overline{\mathbf{q}}_{i}\right\rangle_{\times, \partial \Omega_{i}}+\left\langle\gamma_{\mathrm{D}}^{i} \cdot \mathbf{S L}_{\boldsymbol{\imath}}^{j}\left(\mathbf{v}_{j}\right), \overline{\mathbf{v}}_{i}\right\rangle_{\times, \partial \Omega_{i}} \\
& +\sum_{j=1}^{n}\left\langle\left\{\gamma_{\mathrm{D}}^{j}\right\} \cdot \mathbf{D L}_{\boldsymbol{\imath}}^{j}\left(\mathbf{v}_{j}\right), \overline{\mathbf{v}}_{j}\right\rangle_{\times, \partial \Omega_{j}}+\left\langle\left\{\gamma_{\mathrm{D}}^{j}\right\} \cdot \mathbf{D} \mathbf{L}_{\boldsymbol{\imath}}^{j}\left(\mathbf{q}_{j}\right), \overline{\mathbf{q}}_{j}\right\rangle_{\times, \partial \Omega_{j}} \\
& +\sum_{i=1}^{n} \sum_{\substack{j=1 \\
j \neq i}}^{n}\left\langle\gamma_{\mathrm{D}}^{i} \cdot \mathbf{D L}_{\boldsymbol{\imath}}^{j}\left(\mathbf{v}_{j}\right), \overline{\mathbf{v}}_{i}\right\rangle_{\times, \partial \Omega_{j}}+\left\langle\gamma_{\mathrm{D}}^{i} \cdot \mathbf{D} \mathbf{L}_{\imath}^{j}\left(\mathbf{q}_{j}\right), \overline{\mathbf{q}}_{i}\right\rangle_{\times, \partial \Omega_{j}} .
\end{aligned}
$$

We already know from [13], Proposition 3.13, or [10], Lemma 12, that the second line in (9.6) is a compact contribution. We are going to show that the third line is a compact contribution as well when either $\mathbf{V} \in \mathcal{Z}(\Gamma)$ or $\mathbf{V} \in \mathcal{N}(\Gamma)$. 


\subsection{Compactness result}

In the sequel, $\gamma_{\mathrm{d}}^{j}: H_{\text {loc }}^{1}\left(\Omega_{j}\right) \rightarrow H^{1 / 2}\left(\Omega_{j}\right)$ will refer to the "pointwise" interior Dirichlet trace operator at the boundary of the subdomain $\Omega_{j}$ i.e. $\gamma_{\mathrm{d}}^{j}(u)=\left.\left(\left.u\right|_{\Omega_{j}}\right)\right|_{\partial \Omega_{j}}$ for any $u \in H_{\mathrm{loc}}^{1}\left(\bar{\Omega}_{j}\right)$. Moreover $\langle\cdot, \cdot\rangle_{\partial \Omega_{j}}$ will refer to the duality pairing between $H^{1 / 2}\left(\partial \Omega_{j}\right)$ and $H^{-1 / 2}\left(\partial \Omega_{j}\right)$.

Proposition 9.3. For any $i, j=1 \ldots n$ such that $i \neq j$, the bilinear form $(\mathbf{u}, \mathbf{v}) \mapsto\left\langle\gamma_{\mathrm{N}}^{i} \cdot \boldsymbol{\Psi}_{\mathbf{\imath}}^{j}(\mathbf{u}), \mathbf{v}\right\rangle_{\times, \partial \Omega_{i}}$, with $\Psi_{\imath}^{j}$ defined in (4.1), is compact when restricted either to $z\left(\partial \Omega_{j}\right) \times \mathcal{Z}\left(\partial \Omega_{i}\right)$ or $\mathcal{N}\left(\partial \Omega_{j}\right) \times \mathcal{N}\left(\partial \Omega_{i}\right)$.

Proof. Both statements can be obtained by a straightforward adaptation of the proof of Proposition 3.13 in [13]. We illustrate this by providing the proof of the first statement. The proof of the second statement is obtained by the same adaptation process.

Choose an arbitrary $(\mathbf{u}, \mathbf{v}) \in \mathcal{Z}\left(\partial \Omega_{j}\right) \times \mathcal{Z}\left(\partial \Omega_{i}\right)$. Recall that $\Delta \boldsymbol{\Psi}_{\imath}^{j}(\mathbf{u})=\boldsymbol{\Psi}_{\imath}^{j}(\mathbf{u})$ in $\mathbb{R}^{3} \backslash \bar{\Omega}_{j}$, so that $\operatorname{curl}\left(\operatorname{curl} \boldsymbol{\Psi}_{\boldsymbol{\imath}}^{j}(\mathbf{u})\right)=\nabla\left(\operatorname{div} \boldsymbol{\Psi}_{\boldsymbol{\imath}}^{j}(\mathbf{u})\right)-\boldsymbol{\Psi}_{\boldsymbol{\imath}}^{j}(\mathbf{u})$. Applying Green's formula (2.2), we obtain

$$
\begin{aligned}
\left\langle\gamma_{\mathrm{N}}^{i} \cdot \boldsymbol{\Psi}_{\mathbf{\imath}}^{j}(\mathbf{u}), \mathbf{v}\right\rangle_{\times, \partial \Omega_{i}}= & -\int_{\Omega_{i}} \boldsymbol{\Psi}_{\mathbf{\imath}}^{j}(\mathbf{u}) \cdot \boldsymbol{\Phi}^{i}(\mathbf{v})-\operatorname{curl} \boldsymbol{\Psi}_{\mathbf{\imath}}^{j}(\mathbf{u}) \cdot \mathbf{c u r l} \boldsymbol{\Phi}^{i}(\mathbf{v}) \mathrm{d} \mathbf{x} \\
& -\int_{\Omega_{i}} \operatorname{div} \boldsymbol{\Psi}_{\boldsymbol{\imath}}^{j}(\mathbf{u}) \cdot \operatorname{div} \boldsymbol{\Phi}^{i}(\mathbf{v}) \mathrm{d} \mathbf{x}+\int_{\partial \Omega_{i}} \gamma_{\mathrm{d}}^{i} \Psi_{\boldsymbol{\imath}}^{j}\left(\operatorname{div} \partial \Omega_{j} \mathbf{u}\right) \mathbf{n}_{i} \cdot \boldsymbol{\Phi}^{i}(\mathbf{v}) \mathrm{d} \sigma \\
\left|\left\langle\gamma_{\mathrm{N}}^{i} \cdot \boldsymbol{\Psi}_{\mathbf{\imath}}^{j}(\mathbf{u}), \mathbf{v}\right\rangle_{\times, \partial \Omega_{i}}\right| \leq & \left\|\boldsymbol{\Phi}^{i}(\mathbf{v})\right\|_{\boldsymbol{H}^{1}\left(\Omega_{i}\right)}\left[\left\|\boldsymbol{\Psi}_{\mathbf{\imath}}^{j}(\mathbf{u})\right\|_{\boldsymbol{H}^{1}\left(\Omega_{i}\right)}+\left\|\gamma_{\mathrm{d}}^{i} \Psi_{\mathbf{\imath}}^{j}\left(\operatorname{div} \partial \Omega_{j} \mathbf{u}\right)\right\|_{L^{2}\left(\partial \Omega_{i}\right)}\right] \\
\leq & C\|\mathbf{v}\|_{\boldsymbol{H}^{-1 / 2}\left(\operatorname{div}, \partial \Omega_{i}\right)}\left[\|\mathbf{u}\|_{\boldsymbol{H}_{\times}^{-1 / 2}\left(\partial \Omega_{j}\right)}+\left\|\gamma_{\mathrm{d}}^{i} \Psi_{\boldsymbol{\imath}}^{j}\left(\operatorname{div} \partial \Omega_{j} \mathbf{u}\right)\right\|_{L^{2}\left(\partial \Omega_{i}\right)}\right] .
\end{aligned}
$$

It is well known that $\gamma_{\mathrm{d}}^{i} \Psi_{\boldsymbol{i}}^{j}$ continuously maps $H^{-1 / 2}\left(\partial \Omega_{j}\right)$ into $H^{+1 / 2}\left(\partial \Omega_{i}\right)$, and since the embedding $H^{1 / 2}\left(\partial \Omega_{i}\right) \hookrightarrow L^{2}\left(\partial \Omega_{i}\right)$ is compact, the operator $\gamma_{\mathrm{d}}^{i} \cdot \Psi_{\mathbf{\imath}}^{j} \cdot \operatorname{div}_{\partial \Omega_{j}}$ compactly maps $\boldsymbol{H}^{-1 / 2}\left(\operatorname{div}, \partial \Omega_{j}\right)$ into $L^{2}\left(\partial \Omega_{i}\right)$. Besides, recall that $\boldsymbol{Z}\left(\partial \Omega_{j}\right)$ is compactly embedded in $\boldsymbol{H}_{\times}^{-1 / 2}\left(\partial \Omega_{j}\right)$. This leads to the conclusion.

Denote by $\mathrm{S}: \widehat{\mathbb{H}}(\Gamma)^{2} \rightarrow \mathbb{C}$ the bilinear form in the first line of (9.6). According to Proposition 9.3 there exists a compact operator $\mathrm{K}_{6}: \widehat{\mathbb{H}}(\Gamma) \rightarrow \widehat{\mathbb{H}}(\Gamma)$ such that $\forall \mathbf{U}=\mathbf{U}^{\boldsymbol{Z}}+\mathbf{U}^{\mathcal{N}} \in \widehat{\mathbb{H}}(\Gamma)$ we have

$$
\begin{aligned}
& \Re e\left\{\widehat{\mathrm{B}}\left(\left(\mathrm{R}_{\imath}+\mathrm{K}_{6}\right) \mathbf{U}, \Xi(\mathbf{U})\right)\right\}=\Re e\left\{\mathrm{~S}\left(\mathbf{U}^{\mathcal{N}}, \overline{\mathbf{U}^{\mathcal{N}}}\right)-\mathrm{S}\left(\mathbf{U}^{z}, \overline{\mathbf{U}^{\mathcal{Z}}}\right)\right\} \\
& \text { where } \quad \mathrm{S}(\mathbf{V}, \overline{\mathbf{V}}):=\sum_{i=1}^{n} \sum_{j=1}^{n}\left\langle\gamma_{\mathrm{D}}^{i} \cdot \mathbf{S L}_{\boldsymbol{\imath}}^{j}\left(\mathbf{q}_{j}\right), \overline{\mathbf{q}}_{i}\right\rangle_{\times, \partial \Omega_{i}}+\left\langle\gamma_{\mathrm{D}}^{i} \cdot \mathbf{S L}_{\boldsymbol{\imath}}^{j}\left(\mathbf{v}_{j}\right), \overline{\mathbf{v}}_{i}\right\rangle_{\times, \partial \Omega_{i}} .
\end{aligned}
$$

\subsection{Generalized Gårding inequality for the single layer potential}

Identity (9.7) clearly shows that it is sufficient to prove positivity properties of the bilinear form $\mathrm{S}(,$,$) in$ order to prove (9.3). Since $\mathbf{S L}_{\imath}^{j}$ involves the potential operators $\Psi_{\imath}^{j}, \boldsymbol{\Psi}_{\imath}^{j}$, we derive positivity result form bilinear forms induced by these potentials.

\section{Proposition 9.4.}

$$
\begin{gathered}
\Re e\left\{\sum_{i=1}^{n} \sum_{j=1}^{n}\left\langle\gamma_{\mathrm{d}}^{i} \cdot \Psi_{\imath}^{j}\left(q_{j}\right), \bar{q}_{i}\right\rangle_{\partial \Omega_{i}}\right\} \geq 0 \quad \forall\left(q_{j}\right) \in \prod_{j=1}^{n} H^{-\frac{1}{2}}\left(\partial \Omega_{j}\right), \\
\Re e\left\{\sum_{i=1}^{n} \sum_{j=1}^{n}\left\langle\gamma_{\mathrm{D}}^{i} \cdot \Psi_{\imath}^{j}\left(\mathbf{p}_{j}\right), \overline{\mathbf{p}}_{i}\right\rangle_{\times, \partial \Omega_{i}}\right\} \geq 0 \quad \forall\left(\mathbf{p}_{j}\right) \in \prod_{j=1}^{n} \mathcal{N}\left(\partial \Omega_{j}\right) .
\end{gathered}
$$


Proof. We only prove the second identity, since the first one can be proved in a completely similar manner. Consider some $\left(\mathbf{p}_{j}\right) \in \Pi_{j=1}^{n} \mathcal{N}\left(\partial \Omega_{j}\right)$, and observe that $\boldsymbol{\Psi}_{\boldsymbol{\imath}}^{j}\left(\mathbf{p}_{j}\right)=\mathbf{S L}_{\boldsymbol{\imath}}^{j}\left(\mathbf{p}_{j}\right)$ since $\operatorname{div}_{\partial \Omega_{j}}\left(\mathbf{p}_{j}\right)=0$. Using $\left[\boldsymbol{\gamma}_{\mathrm{N}}^{i}\right]$. $\mathbf{S L}_{\boldsymbol{\imath}}^{i}\left(\mathbf{p}_{i}\right)=\mathbf{p}_{i}$ according to (4.2), each term in the left hand side of the desired inequality can be rewritten in the following manner

$$
\left\langle\gamma_{\mathrm{D}}^{i} \cdot \boldsymbol{\Psi}_{\boldsymbol{\imath}}^{j}\left(\mathbf{p}_{j}\right), \overline{\mathbf{p}}_{i}\right\rangle_{\times, \partial \Omega_{i}}=\left\langle\gamma_{\mathrm{D}}^{i} \cdot \mathbf{S L}_{\boldsymbol{\imath}}^{j}\left(\mathbf{p}_{j}\right),\left[\gamma_{\mathrm{N}}^{i}\right] \cdot \overline{\mathbf{S L}_{\boldsymbol{\imath}}^{i}\left(\mathbf{p}_{i}\right)}\right\rangle_{\times, \partial \Omega_{i}} .
$$

Applying Green's formula (2.2) both in $\Omega_{i}$ and $\mathbb{R}^{3} \backslash \bar{\Omega}_{i}$, and taking account that $\mathbf{S L}_{\boldsymbol{\imath}}^{j}\left(\mathbf{p}_{j}\right) \in \boldsymbol{H}\left(\mathbf{c u r l}, \mathbb{R}^{3}\right)$ so that $\boldsymbol{\gamma}_{\mathrm{D}}^{i} \cdot \mathbf{S L}_{\boldsymbol{\imath}}^{j}\left(\mathbf{p}_{j}\right)=\boldsymbol{\gamma}_{\mathrm{D}, c}^{i} \cdot \mathbf{S L}_{\boldsymbol{\imath}}^{j}\left(\mathbf{p}_{j}\right) \forall i, j=1 \ldots n$, and $\operatorname{curl}^{2}\left(\mathbf{S L}_{\boldsymbol{\imath}}^{i}\left(\mathbf{p}_{i}\right)\right)+\mathbf{S L}_{\boldsymbol{\imath}}^{i}\left(\mathbf{p}_{i}\right)=\mathbf{0}$ in $\Omega_{i} \cup\left(\mathbb{R}^{3} \backslash \bar{\Omega}_{i}\right)$ for all $i=1 \ldots n$, we have

$$
\left\langle\gamma_{\mathrm{D}}^{i} \cdot \boldsymbol{\Psi}_{\imath}^{j}\left(\mathbf{p}_{j}\right), \overline{\mathbf{p}}_{i}\right\rangle_{\times, \partial \Omega_{i}}=\int_{\mathbb{R}^{3}} \operatorname{curl}\left(\mathbf{S L}_{\imath}^{j}\left(\mathbf{p}_{j}\right)\right) \cdot \operatorname{curl}\left(\overline{\mathbf{S L}_{\imath}^{i}\left(\mathbf{p}_{i}\right)}\right)+\mathbf{S L}_{\imath}^{j}\left(\mathbf{p}_{j}\right) \cdot \overline{\mathbf{S L}_{\imath}^{i}\left(\mathbf{p}_{i}\right)} \mathrm{d} \mathbf{x} .
$$

Summing over $i, j=1 \ldots n$, we obtain

$$
\sum_{i=1}^{n} \sum_{j=1}^{n}\left\langle\boldsymbol{\gamma}_{\mathrm{D}}^{i} \cdot \boldsymbol{\Psi}_{\mathbf{\imath}}^{j}\left(\mathbf{p}_{j}\right), \overline{\mathbf{p}}_{i}\right\rangle_{\times, \partial \Omega_{i}}=\left\|\sum_{j=1}^{n} \mathbf{S L}_{\mathbf{\imath}}^{j}\left(\mathbf{p}_{j}\right)\right\|_{\boldsymbol{H}\left(\mathbf{c u r l}, \mathbb{R}^{3}\right)}^{2} \geq 0 .
$$

This yields the assertion.

From the proposition above, we can deduce positivity properties of the bilinear form $\mathrm{S}(,$,$) up to some$ compact perturbation.

Corollary 9.5. There exists a compact operator $\mathrm{K}_{7}: \widehat{\mathbb{H}}(\Gamma) \rightarrow \widehat{\mathbb{H}}(\Gamma)$ such that

$$
\begin{array}{r}
\Re e\{\mathrm{~S}(\mathbf{V}, \overline{\mathbf{V}})\} \geq 0, \forall \mathbf{V} \in \mathcal{N}(\Gamma) . \\
\Re e\left\{-\mathrm{S}(\mathbf{V}, \overline{\mathbf{V}})+\widehat{\mathrm{B}}\left(\mathrm{K}_{7} \mathbf{V}, \overline{\mathbf{V}}\right)\right\} \\
\geq 0, \forall \mathbf{V} \in \mathbf{z}(\Gamma) .
\end{array}
$$

Proof. Assume first that $\mathbf{V}=\left(\mathbf{v}_{j}, \mathbf{q}_{j}\right)_{j=1 \ldots n}^{\top} \in \mathcal{N}(\Gamma)$. Then we have $\mathbf{S L}_{\boldsymbol{\imath}}^{j}\left(\mathbf{v}_{j}\right)=\boldsymbol{\Psi}_{\boldsymbol{\imath}}^{j}\left(\mathbf{v}_{j}\right)$ and $\mathbf{S L}_{\imath}^{j}\left(\mathbf{q}_{j}\right)=\boldsymbol{\Psi}_{\imath}^{j}\left(\mathbf{q}_{j}\right)$. Hence in this case, the proof is a straightforward application of Proposition 9.4, since

$$
\Re e\{\mathrm{~S}(\mathbf{V}, \overline{\mathbf{V}})\}=\Re e\left\{\sum_{i=1}^{n} \sum_{j=1}^{n}\left\langle\gamma_{\mathrm{D}}^{i} \cdot \boldsymbol{\Psi}_{\boldsymbol{\imath}}^{j}\left(\mathbf{v}_{j}\right), \overline{\mathbf{v}}_{i}\right\rangle_{\times, \partial \Omega_{i}}+\left\langle\gamma_{\mathrm{D}}^{i} \cdot \boldsymbol{\Psi}_{\boldsymbol{\imath}}^{j}\left(\mathbf{v}_{j}\right), \overline{\mathbf{v}}_{i}\right\rangle_{\times, \partial \Omega_{i}}\right\} .
$$

Now let us consider $\mathbf{V}=\left(\mathbf{v}_{j}, \mathbf{q}_{j}\right)_{j=1 \ldots n}^{\top} \in \boldsymbol{Z}(\Gamma)$ so as to prove the second inequality. Applying an integration by parts formula for surfaces on each boundary $\partial \Omega_{i}$, we have the expression

$$
\begin{aligned}
-\mathrm{S}(\mathbf{V}, \overline{\mathbf{V}})= & \sum_{i=1}^{n} \sum_{j=1}^{n}\left\langle\gamma_{\mathrm{d}}^{i} \cdot \Psi_{\boldsymbol{\imath}}^{j}\left(\operatorname{div}_{\partial \Omega_{j}} \mathbf{v}_{j}\right), \overline{\operatorname{div} \partial \Omega_{i} \mathbf{v}_{i}}\right\rangle_{\times, \partial \Omega_{i}} \\
& +\sum_{i=1}^{n} \sum_{j=1}^{n}\left\langle\gamma_{\mathrm{d}}^{i} \cdot \Psi_{\boldsymbol{\imath}}^{j}\left(\operatorname{div}_{\partial \Omega_{j}} \mathbf{q}_{j}\right), \overline{\operatorname{div} \partial \Omega_{i} \mathbf{q}_{i}}\right\rangle_{\times, \partial \Omega_{i}} \\
& -\sum_{i=1}^{n} \sum_{j=1}^{n}\left\langle\gamma_{\mathrm{D}}^{i} \cdot \boldsymbol{\Psi}_{\boldsymbol{\imath}}^{j}\left(\mathbf{v}_{j}\right), \overline{\mathbf{v}}_{i}\right\rangle_{\times, \partial \Omega_{i}}+\left\langle\boldsymbol{\gamma}_{\mathrm{D}}^{i} \cdot \boldsymbol{\Psi}_{\boldsymbol{\imath}}^{j}\left(\mathbf{q}_{j}\right), \overline{\mathbf{q}_{i}}\right\rangle_{\times, \partial \Omega_{i}} .
\end{aligned}
$$

Since the operator $\boldsymbol{\gamma}_{\mathrm{D}}^{i} \cdot \boldsymbol{\Psi}_{\boldsymbol{\imath}}^{j}: \boldsymbol{H}_{\times}^{-1 / 2}\left(\partial \Omega_{j}\right) \rightarrow \boldsymbol{H}_{\times}^{+1 / 2}\left(\partial \Omega_{i}\right)$ is continuous for any $i, j=1 \ldots n$, and since $z\left(\partial \Omega_{j}\right)$ is compactly embedded in $\boldsymbol{H}_{\times}^{-1 / 2}\left(\partial \Omega_{j}\right)$, we conclude that the third line in (9.9) only contains compact contributions. As a consequence, the first inequality provided by Proposition 9.4 leads to the conclusion of the proof. 
We now have all the necessary ingredients to establish a generalized Gårding inequality for the operator $\widehat{\mathrm{A}}_{\kappa, \mu}$. Indeed in Sections $9.2-9.4$, we saw that such an inequality holds if there exists a compact operator $\mathrm{K}: \widehat{\mathbb{H}}(\Gamma) \rightarrow \widehat{\mathbb{H}}(\Gamma)$ such that

$$
\Re e\left\{\widehat{\mathrm{B}}(\mathrm{K} \mathbf{U}, \Xi(\mathbf{U}))+\mathrm{S}\left(\mathbf{U}^{\mathcal{N}}, \overline{\mathbf{U}^{\mathcal{N}}}\right)-\mathrm{S}\left(\mathbf{U}^{\mathcal{Z}}, \overline{\mathbf{U}^{\mathcal{Z}}}\right)\right\} \geq 0 \quad \forall \mathbf{U}=\mathbf{U}^{\mathcal{N}}+\mathbf{U}^{\mathcal{Z}} \in \widehat{\mathbb{H}}(\Gamma) .
$$

Corollary 9.5 shows that such an estimate indeed holds for some compact operator K. This proves the following main result of this section.

Theorem 9.6 (coercivity of multi-trace operator). There exists a compact operator $\mathrm{K}: \widehat{\mathbb{H}}(\Gamma) \rightarrow \widehat{\mathbb{H}}(\Gamma)$ such that the following generalized Gårding inequality holds

$$
\Re e\left\{\widehat{\mathrm{B}}\left(\left(\widehat{\mathrm{A}}_{\kappa, \mu}+\mathrm{K}\right) \mathbf{U}, \Xi(\mathbf{U})\right)\right\} \geq 0 \quad \forall \mathbf{U} \in \widehat{\mathbb{H}}(\Gamma) .
$$

From this theorem we conclude in particular that $\widehat{\mathrm{A}}_{\kappa, \mu}: \widehat{\mathbb{H}}(\Gamma) \rightarrow \widehat{\mathbb{H}}(\Gamma)$ is a Fredholm operator with index zero. It actually is an isomorphism.

Corollary 9.7. The operator $\widehat{\mathrm{A}}_{\kappa, \mu}: \widehat{\mathbb{H}}(\Gamma) \rightarrow \widehat{\mathbb{H}}(\Gamma)$ is an isomorphism.

Proof. According to Fredholm alternative, we only need to prove that $\widehat{\mathrm{A}}_{\kappa, \mu}$ is one-to-one. Set $\widehat{\mathbf{F}}=0$. Then the problem

$$
\text { Find } \mathbf{U} \in \widehat{\mathbb{H}}(\Gamma) \text { such that } \widehat{\mathrm{B}}\left(\widehat{\mathrm{A}}_{\kappa, \mu} \mathbf{U}, \mathbf{V}\right)=\widehat{\mathrm{B}}(\widehat{\mathbf{F}}, \mathbf{V}), \quad \forall \mathbf{V} \in \widehat{\mathbb{H}}(\Gamma)
$$

actually fits the assumptions of Theorem 8.1. As a consequence, it admits a unique solution that is $\mathbf{U}=0$. Hence $\widehat{\mathrm{A}}_{\kappa, \mu}$ is one-to-one.

\section{GALERKIN BOUNDARY ELEMENT DisCRETIZATION}

We briefly address the Galerkin discretization of Formulation (8.4). Proof of optimal rate of convergence under reasonable discretization process relies on known results. Indeed, in Section 9 we have just proved that formulation (8.4) fits the framework of [6], as it satisfies Assumption 1 of this article. As a consequence, we can state the following result as a direct application of [6], Theorem 3.7.

Proposition 10.1. Let $\left(\widehat{\mathbb{H}}_{h}\right)_{0<h<1}$ with $\widehat{\mathbb{H}}_{h} \subset \widehat{\mathbb{H}}(\Gamma)$ be any family of finite dimensional subspace that satisfies two assumptions

- (CAS) property: the family $\left(\widehat{\mathbb{H}}_{h}\right)$ has the complete approximation property:

$$
\lim _{h \rightarrow 0} \inf _{\mathbf{U}_{h} \in \widehat{\mathbb{H}}_{h}}\left\|\mathbf{U}-\mathbf{U}_{h}\right\|=0 \quad \forall \mathbf{U} \in \widehat{\mathbb{H}}(\Gamma)
$$

- (GAP) property: the family $\left(\widehat{\mathbb{H}}_{h}\right)$ admits a decomposition that satisfies the gap property: there exists subspaces $\boldsymbol{Z}_{h} \subset \widehat{\mathbb{H}}_{h}$ and $\mathcal{N}_{h} \subset \widehat{\mathbb{H}}_{h}$ such that

$$
\sup _{\mathbf{U}_{h} \in \mathcal{Z}_{h}} \inf _{\mathbf{U} \in \mathcal{Z}(\Gamma)} \frac{\left\|\mathbf{U}-\mathbf{U}_{h}\right\|}{\left\|\mathbf{U}_{h}\right\|}+\sup _{\mathbf{U}_{h} \in \mathcal{N}_{h}} \inf _{\mathbf{U} \in \mathcal{N}(\Gamma)} \frac{\left\|\mathbf{U}-\mathbf{U}_{h}\right\|}{\left\|\mathbf{U}_{h}\right\|} \underset{h \rightarrow 0}{\longrightarrow} 0 .
$$

Under the above assumptions, there exists $c_{0}, h_{0}>0$ such that, the following uniform discrete inf - sup condition is satisfied

$$
\inf _{\mathbf{U}_{h} \in \widehat{\mathbb{H}}_{h}} \sup _{\mathbf{V}_{h} \in \widehat{\mathbb{H}}_{h}} \frac{\Re e\left\{\widehat{\mathrm{B}}\left(\widehat{\mathrm{A}}_{\kappa, \mu} \mathbf{U}_{h}, \mathbf{V}_{h}\right)\right\}}{\left\|\mathbf{U}_{h}\right\|\left\|\mathbf{V}_{h}\right\|}>c_{0} \quad \forall h \in\left(0, h_{0}\right) .
$$


Recall that $\widehat{\mathrm{B}}\left(\widehat{\mathrm{A}}_{\kappa, \mu} \mathbf{U}, \mathbf{V}\right)=\widehat{\mathrm{B}}\left(\widehat{\mathrm{A}}_{\kappa, \mu} \mathbf{V}, \mathbf{U}\right) \forall \mathbf{U}, \mathbf{V} \in \widehat{\mathbb{H}}(\Gamma)$ according to Proposition 8.2. As a consequence, see [34], Theorem 4.2.1, if the discretization process obeys the assumptions of Proposition 10.1, the corresponding Galerkin approximation is quasi-optimally convergent.

Proposition 10.2. Consider any $\mathbf{F} \in \widehat{\mathbb{H}}(\Gamma)$. Consider a family of finite dimensional subspaces $\left(\widehat{\mathbb{H}}_{h}\right)_{0<h<1}$ with $\widehat{\mathbb{H}}_{h} \subset \widehat{\mathbb{H}}(\Gamma)$, that satisfy the $(C A S)$ and $(G A P)$ assumptions of Proposition 10.1. Consider the problem

$$
\text { Find } \mathbf{U}_{h} \in \widehat{\mathbb{H}}_{h} \quad \text { such that } \widehat{\mathrm{B}}\left(\widehat{\mathrm{A}}_{\kappa, \mu} \mathbf{U}_{h}, \mathbf{V}_{h}\right)=\widehat{\mathrm{B}}\left(\mathbf{F}, \mathbf{V}_{h}\right) \quad \forall \mathbf{V}_{h} \in \widehat{\mathbb{H}}_{h} \text {. }
$$

Then there exists $h_{0}>0$ such that problem (10.1) admits a unique solution for all $h \in\left(0, h_{0}\right)$. Moreover if $\mathbf{U}$ refers to the unique solution to problem (8.4), then there exists $C>0$ independent of $h$ such that

$$
\left\|\mathbf{U}-\mathbf{U}_{h}\right\| \leq C \inf _{\mathbf{V}_{h} \in \mathbb{\mathbb { H }}_{h}}\left\|\mathbf{U}-\mathbf{V}_{h}\right\| \quad \forall h \in\left(0, h_{0}\right) .
$$

We point out that, to obtain a discrete variational space $\widehat{\mathbb{H}}_{h}$ fulfilling the assumptions of Proposition 10.1, it suffices to define it as a cartesian product $\widehat{\mathbb{H}}_{h}=\mathbb{H}_{h}\left(\partial \Omega_{1}\right) \times \cdots \times \mathbb{H}_{h}\left(\partial \Omega_{n}\right)$, where each $\widehat{\mathbb{H}}_{h}\left(\partial \Omega_{j}\right)$ satisfies $(\mathrm{CAS})$ and $(\mathrm{GAP})$ relatively to $\mathbb{H}\left(\partial \Omega_{j}\right)=\left(\mathcal{Z}\left(\partial \Omega_{j}\right)\right)^{2} \oplus\left(\mathcal{N}\left(\partial \Omega_{j}\right)\right)^{2}$.

For example, one may take $\widehat{\mathbb{H}}_{h}\left(\partial \Omega_{j}\right)$ as Raviart-Thomas or Brezzi-Douglas-Marini finite elements constructed on a regular family of triangulation of $\partial \Omega_{j}$. According to [13], Section 4, such discretizations satisfy the (CAS) and (GAP) properties local to $\partial \Omega_{j}$.

Finally let us underline one comfortable aspect of formulation (10.1) as regards implementation: it does not require any particular treatment of triple junctions (i.e. points of $\Gamma$ where three or more subdomains abut). Moreover this formulation permits us to choose the trial and test spaces on different boundaries of subdomains completely independently.

\section{CALderón PRECONDITIONing}

Eventually, the possibility for Calderón preconditioning provides the chief rationale for our investigating the new multi-trace formulation (8.4). It seems that this possibility is elusive for the single-trace formulation (5.2), unless all bounded subdomain are separated, see [21,41].

However, this is a crucial observation, if Calderón identities for the single-trace formulation are available for separated subdomains, we may again resort to the gap idea presented in Section 5 to transfer them to our new multi-trace formulation. Again, to explain this, we zero in on the model setting with two bounded subdomains $(n=2)$ and assume $\mu_{0}=\mu_{1}=\mu_{2}$, see Figure 2 in Section 5.

For the Calderón projector associated with $\Omega_{0}$ the standard Calderón identity $\left(\frac{1}{2} \operatorname{Id}+\mathrm{C}_{\kappa_{0}}^{0}\right)^{2}=\frac{1}{2} \operatorname{Id}+\mathrm{C}_{\kappa_{0}}^{0}$ when expressed in terms of traces on the subdomain boundaries $\partial \Omega_{1}$ and $\partial \Omega_{2}$ as in (5.5), immediately yields

$$
\mathrm{R}_{\kappa_{0}}^{1,2} \mathrm{R}_{\kappa_{0}}^{2,1}=\mathrm{R}_{\kappa_{0}}^{2,1} \mathrm{R}_{\kappa_{0}}^{1,2}=0, \quad \mathrm{R}_{\kappa_{0}}^{2,1} \mathrm{C}_{\kappa_{0}}^{1}+\mathrm{C}_{\kappa_{0}}^{2} \mathrm{R}_{\kappa_{0}}^{2,1}=0, \quad \mathrm{R}_{\kappa_{0}}^{1,2} \mathrm{C}_{\kappa_{0}}^{2}+\mathrm{C}_{\kappa_{0}}^{1} \mathrm{R}_{\kappa_{0}}^{1,2}=0
$$

Using these identities along with $\left(2 \mathrm{C}_{\kappa}^{1}\right)^{2}=\mathrm{Id}$ and $\left(2 \mathrm{C}_{\kappa}^{2}\right)^{2}=\mathrm{Id}$ for any $\kappa \in \mathbb{R}_{+} \backslash\{0\}$, we find for the multi-trace boundary integral operator from (5.7)

$$
\left(\begin{array}{cc}
\mathrm{C}_{\kappa}^{1}+\mathrm{C}_{\kappa}^{1} & \mathrm{R}_{\kappa}^{1,2} \\
\mathrm{R}_{\kappa}^{2,1} & \mathrm{C}_{\kappa}^{2}+\mathrm{C}_{\kappa}^{2}
\end{array}\right)^{2}=\mathrm{Id} \quad \text { on } \quad \widehat{\mathbb{H}}(\Gamma),
$$

for any $\kappa \in \mathbb{C}$. Recalling from Section 9.3 that a change of the wave number amounts to a compact perturbation (recall that we assume $\mu_{0}=\mu_{1}=\mu_{2}$ here), we see that there is a compact operator $\mathrm{K}: \widehat{\mathbb{H}} \rightarrow \widehat{\mathbb{H}}$ such that

$$
\left(\begin{array}{cc}
2 \mathrm{C}_{\kappa}^{1} & \mathrm{R}_{\kappa}^{1,2} \\
\mathrm{R}_{\kappa}^{2,1} & 2 \mathrm{C}_{\kappa}^{2}
\end{array}\right)\left(\begin{array}{cc}
\mathrm{C}_{\kappa_{1}}^{1}+\mathrm{C}_{\kappa_{0}}^{1} & \mathrm{R}_{\kappa_{0}}^{1,2} \\
\mathrm{R}_{\kappa_{0}}^{2,1} & \mathrm{C}_{\kappa_{1}}^{2}+\mathrm{C}_{\kappa_{0}}^{2}
\end{array}\right)=\mathrm{Id}+\mathrm{K} .
$$


In words, the first left boundary integral operator in (11.3) is a preconditioning operator for the multi-trace boundary integral operator that has the effect of making the spectrum of the product operator cluster around 1.

From these considerations it should become clear how the Calderón identity for the general multi-trace operator (8.2) will read. We state it in the following result. The proof, that we do not provide, is exactly the same as for Theorem 11.1 in [19].

Theorem 11.1 (Calderón identity for multi-trace boundary integral operator). If $\kappa_{0}=\kappa_{1}=\ldots=\kappa_{n}$ and $\mu_{0}=\mu_{1}=\ldots=\mu_{n}$ with $\kappa_{0}, \mu_{0} \in(0,+\infty)$, then $\left(\widehat{\mathrm{A}}_{\kappa, \mu}\right)^{2}=\mathrm{Id}$.

This paves the way of applying the powerful technique of operator preconditioning [25] to the discrete multi-trace formulation (10.1). What is needed is another family of finite dimensional subspaces $\left(\widetilde{\mathbb{H}}_{h}\right)_{0<h<1}$ that

- gives rise to an $h$-uniformly stable discretization of (10.1) by meeting the requirements stated in Section 10;

- supports a stable discrete duality pairing in the sense that, for some $h_{0}>0$,

$$
\inf _{\mathbf{U}_{h} \in \widehat{\mathbb{H}}_{h}} \sup _{\mathbf{V}_{h} \in \widetilde{\mathbb{H}}_{h}} \frac{\Re e\left\{\widehat{\mathrm{B}}\left(\widehat{\mathrm{A}}_{\kappa, \mu} \mathbf{U}_{h}, \mathbf{V}_{h}\right)\right\}}{\left\|\mathbf{U}_{h}\right\|\left\|\mathbf{V}_{h}\right\|}>c_{d} \quad \forall h \in\left(0, h_{0}\right) .
$$

This condition entails $\operatorname{dim} \widehat{\mathbb{H}}=\operatorname{dim} \widetilde{\mathbb{H}}$.

Then [25], Theorem 2.1, tells us that the spectral condition number of the product matrix $\boldsymbol{D}_{h}^{-1} \boldsymbol{B}_{h} \boldsymbol{D}_{h}^{-\top} \boldsymbol{A}_{h}$ is bounded independently of $h$, which renders $\boldsymbol{D}_{h}^{-1} \boldsymbol{B}_{h} \boldsymbol{D}_{h}^{-\top}$ an asymptotically optimal preconditioner. Here,

- $\boldsymbol{A}_{h}$ is the $\widehat{\mathbb{H}}_{h}$-Galerkin matrix for the multi-trace variational problem (8.4);

- $\boldsymbol{B}_{h}$ is the Galerkin matrix for the multi-trace operator with globally constant coefficients $\kappa, \mu$ and with respect to the trial and test spaces $\widetilde{\mathbb{H}}_{h}$;

- $\boldsymbol{D}_{h}$ is the Galerkin matrix for the pairing $\widehat{\mathrm{B}}: \widehat{\mathbb{H}}(\Gamma) \times \widehat{\mathbb{H}}(\Gamma) \rightarrow \mathbb{C}$ discretized on $\widehat{\mathbb{H}}_{h} \times \widetilde{\mathbb{H}}_{h}$.

As discovered by Buffa and Christiansen [7], when Raviart-Thomas boundary elements are used to build $\widehat{\mathbb{H}}_{h}$, the same type of elements on a dual mesh can provide suitable spaces $\widetilde{\mathbb{H}}_{h}$. For details we refer to $[1,7]$.

\section{REFERENCES}

[1] F.P. Andriulli, K. Cools, H. Bagci, F. Olyslager, A. Buffa, S. Christiansen and E. Michielssen, A multiplicative Calderon preconditioner for the electric field integral equation. IEEE Trans. Antennas Propag. 56 (2008) 2398-2412.

[2] H. Bagci, F.P. Andriulli, K. Coolst, F. Olyslager and E. Michielssen, A Calderón multiplicative preconditioner for the combined field integral equation. IEEE Trans. Antennas Propag. 57 (2009) 3387-3392.

[3] A. Bendali, Numerical analysis of the exterior boundary value problem for time harmonic Maxwell equations by a boundary finite element method. Part 2: The discrete problem. Math. Comput. 43 (1984) 47-68.

[4] A. Bendali, M.B. Fares and J. Gay, A boundary-element solution of the Leontovitch problem. IEEE Trans. Antennas Propag. 47 (1999) 1597-1605.

[5] Y. Boubendir, A. Bendali and M.B. Fares, Coupling of a non-overlapping domain decomposition method for a nodal finite element method with a boundary element method. Int. J. Numer. Methods Eng. 73 (2008) 1624-1650.

[6] A. Buffa, Remarks on the discretization of some noncoercive operator with applications to the heterogeneous Maxwell equations. SIAM J. Numer. Anal. 43 (2005) 1-18.

[7] A. Buffa and S.H. Christiansen, A dual finite element complex on the barycentric refinement. Math. Comput. 76 (2007) $1743-1769$.

[8] A. Buffa and P. Ciarlet Jr., On traces for functional spaces related to Maxwell's equations I. An integration by parts formula in Lipschitz polyhedra. Math. Methods Appl. Sci. 24 (2001) 9-30.

[9] A. Buffa and P. Ciarlet Jr., On traces for functional spaces related to Maxwell's equations II. Hodge decompositions on the boundary of Lipschitz polyhedra and applications. Math. Methods Appl. Sci. 24 (2001) 31-48.

[10] A. Buffa and R. Hiptmair, Galerkin boundary element methods for electromagnetic scattering, in Topics in computational wave propagation. Lect. Notes Comput. Sci. Eng. 31 (2003) 83-124.

[11] A. Buffa, M. Costabel and C. Schwab, Boundary element methods for Maxwell's equations on non-smooth domains. Numer. Math. 92 (2002) 679-710. 
[12] A. Buffa, M. Costabel and D. Sheen, On traces for H(curl, $\Omega$ ) in Lipschitz domains. J. Math. Anal. Appl. 276 (2002) 845-867.

[13] A. Buffa, R. Hiptmair, T. von Petersdorff and C. Schwab, Boundary element methods for Maxwell transmission problems in Lipschitz domains. Numer. Math. 95 (2003) 459-485.

[14] Y. Chang and R. Harrington, A surface formulation or characteristic modes of material bodies. IEEE Trans. Antennas Propag. 25 (1977) 789-795.

[15] S.H. Christiansen, Discrete Fredholm properties and convergence estimates for the electric field integral equation. Math. Comput. 73 (2004) 143-167.

[16] S.H. Christiansen and J.-C. Nédélec, Des préconditionneurs pour la résolution numérique des équations intégrales de frontière de l'acoustique. C. R. Acad. Sci. Paris, Sér. I Math. 330 (2000) 617-622.

[17] S.H. Christiansen and J.-C. Nédélec, A preconditioner for the electric field integral equation based on Calderón formulas. SIAM J. Numer. Anal. 40 (2002) 1100-1135.

[18] X. Claeys, A single trace integral formulation of the second kind for acoustic scattering. Seminar of Applied Mathematics, ETH Zürich, Technical Report 2011-14. Submitted to J. Appl. Math. (2011).

[19] X. Claeys and R. Hiptmair, Boundary integral formulation of the first kind for acoustic scattering by composite structures. Report 2011-45, SAM, ETH Zürich (2011)

[20] D. Colton and R. Kress, Inverse acoustic and electromagnetic scattering theory, 2nd edition. Appl. Math. Sci. 93 (1998).

[21] K. Coolst, F.P. Andriulli and F. Olyslager, A Calderón, preconditioned PMCHWT equation, in Proc. of the International Conference on Electromagnetics in Advanced Applications, ICEAA'09. Torino, Italy (2009) 521-524.

[22] M. Costabel, Boundary integral operators on Lipschitz domains: elementary results. SIAM J. Math. Anal. 19 (1988) 613-626.

[23] R. Hiptmair, Finite elements in computational electromagnetism. Acta Numer. 11 (2002) 237-339.

[24] R. Hiptmair, Coupling of finite elements and boundary elements in electromagnetic scattering. SIAM J. Numer. Anal. 41 (2003) 919-944.

[25] R. Hiptmair, Operator preconditioning. Comput. Math. Appl. 52 (2006) 699-706.

[26] R. Hiptmair and C. Jerez-Hanckes, Multiple traces boundary integral formulation for Helmholtz transmission problems. SAM, ETH Zürich, Report 2010-35 (2010).

[27] R. Hiptmair and C. Schwab, Natural boundary element methods for the electric field integral equation on polyhedra. SIAM J. Numer. Anal. 40 (2002) 66-86.

[28] G.C. Hsiao, O. Steinbach and W.L. Wendland, Domain decomposition methods via boundary integral equations, Numerical Analysis VI, Ordinary differential equations and integral equations. J. Comput. Appl. Math. 125 (2000) 521-537.

[29] S. Järvenpää, S.P. Kiminki and P. Ylä-Oijala, Calderon preconditioned surface integral equations for composite objects with junctions. IEEE Trans. Antennas Propag. 59 (2011) 546-554.

[30] U. Langer and O. Steinbach, Boundary element tearing and interconnecting methods. Computing 71 (2003) 205-228.

[31] W. McLean, Strongly elliptic systems and boundary integral equations. Cambridge University Press, Cambridge (2000).

[32] E. Miller and A. Poggio, Computer Techniques for Electromagnetics, in Integral equation solution of three-dimensional scattering problems, Chapter 4, Pergamon, New York (1973) 159-263.

[33] S.M. Rao, D.R. Wilton and A.W. Glisson, Electromagnetic scattering by surfaces of arbitrary shape. IEEE Trans. Antennas Propag. 30 (1986) 409-418.

[34] S.A. Sauter and C. Schwab, Boundary element methods, Springer Series in Comput. Math. 39 (2011).

[35] O. Steinbach and W.L. Wendland, The construction of some efficient preconditioners in the boundary element method. Adv. Comput. Math 9 (1998) 191-216.

[36] O. Steinbach and M. Windisch, Modified combined field integral equations for electromagnetic scattering. SIAM J. Numer. Anal. 47 (2009) 1149-1167.

[37] M.B. Stephanson and J.-F. Lee, Preconditioned electric field integral equation using Calderon identities and dual loop/star basis functions. IEEE Trans. Antennas Propag. 57 (2009) 1274-1279.

[38] T. von Petersdorff, Boundary integral equations for mixed Dirichlet, Neumann and transmission problems. Math. Methods Appl. Sci. 11 (1989) 185-213.

[39] M. Windisch, Boundary element tearing and interconnecting methods for acoustic and electromagnetic scattering. Ph.D. thesis, Graz University of Technology (2010).

[40] T.-K. Wu and L.-L. Tsai, Scattering from arbitrarily-shaped lossy di-electric bodies of revolution. Radio Sci. 12 (1977) 709-718.

[41] S. Yan, J.-M. Jin and Z.-P. Nie, A comparative study of Calderón preconditioners for PMCHWT equations. IEEE Trans. Antennas Propag. 58 (2010) 2375-2383. 